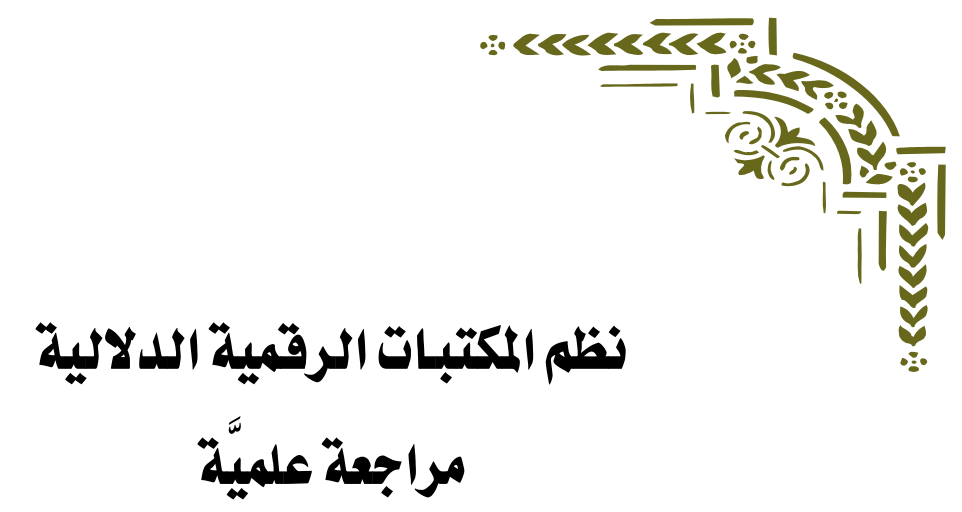

مثار متمداد سامع ز5كي

طالبة دراسات عليا - قسهم علوم المعلومات - جامعة بني سويف

أ.د. عبدالو ممن خراج

أستاذ علم المعلومات - قسم علوم المعلومات - جامعة بني سويف

أ.د. وحاب دوبسف.

أستاذ تكنولوجيا المعلومات - قسهم علوم المعلومات - جامعة بني سويف

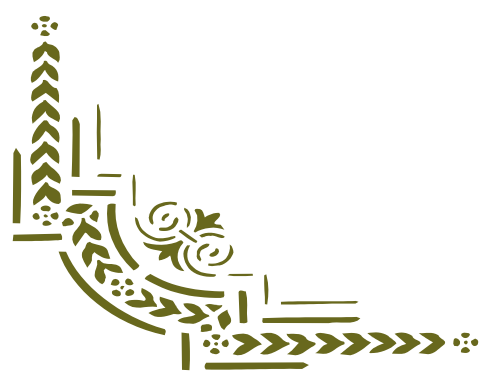

$$
\begin{aligned}
& \text { مج8، ع1، ابريل 2021م } \\
& \text { المجلة المصيرية لعلوم المعلومات }
\end{aligned}
$$




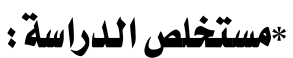

تتناول هذه المراجعة رصيد الإنتاج الفكري في نظم المكتبات الرقميَّة الدلاليَّة،

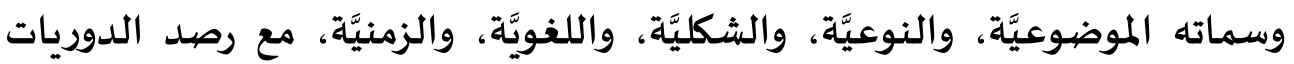

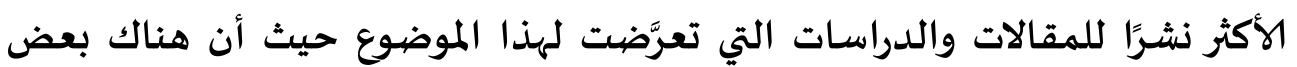
الدراسات التي تناولت جزءًا من موضوع البحث كالويب الدلالي، ومحاولة إبراز

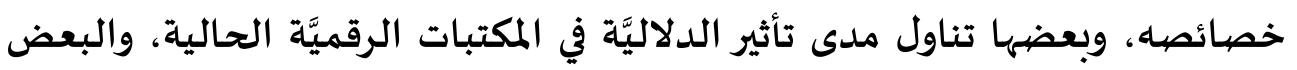

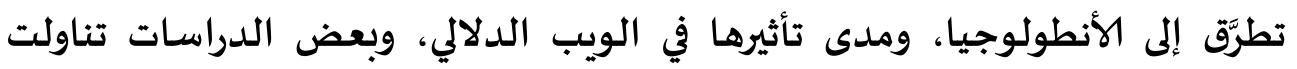

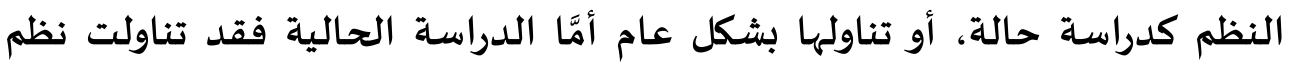

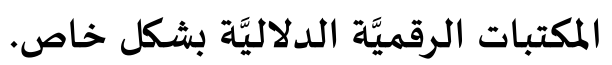

\section{الكلمات المفتاحية}

$$
\text { المكتبات الرقمية الدلالية - الويب الدلالي - المراجعات العلمية }
$$


اهتمَّ العديد من الدراسـات سواء كانت العربيَّة أو الأجنبيَّة بمواكبة الشبكة

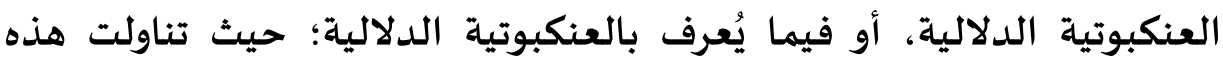

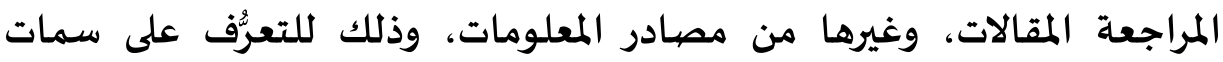
وخصيائص هذا ودراسته دراسـة تقييميَّة، نقديَّة، تحليليَّة.

\section{2}

ترجع اهمية هذه المراجعة التي تنصب على المكتبات الرقمية الدلالية فيما يلي: - الإحاطة بما هو جارٍ من تطورات تقنية العنكبوتية الدلالية بشكل عام، والنظم

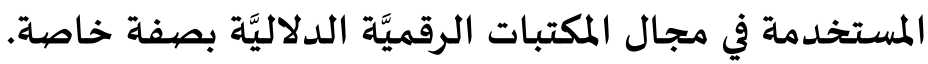
- الإحاطة بما تمَّ تناوله في الإنتاج الفكري المتخصص على مستوى الدراسة،

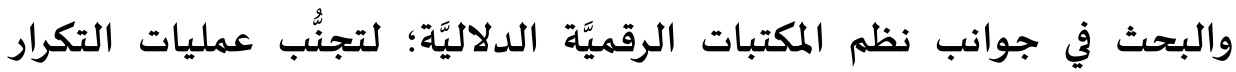
البحوث، والتعرُّف على الموضيوعات الشـاغرة، وتلك التي لم يتمّ تناولها بكثافة على مستوى الدراسات البحثيَّة.

- التعرُف على خصيائص وسمات الإنتاج الفكري المتخصص في موضوع نظم المكتبات الرقميَّة الدلاليَّة.

\section{3}

الندرة البحثية حول وصف خصائص الإنتاج الفكري الخاص بنظم المكتبات

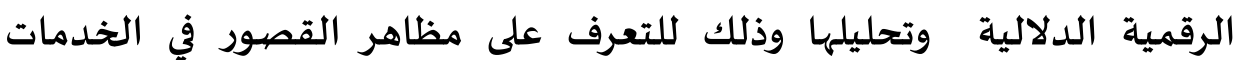

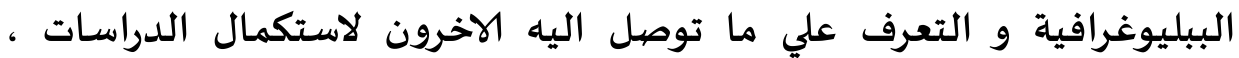

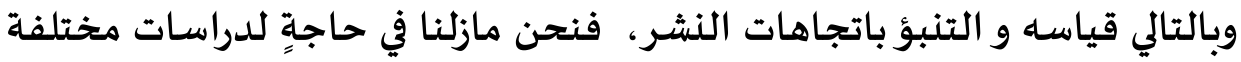

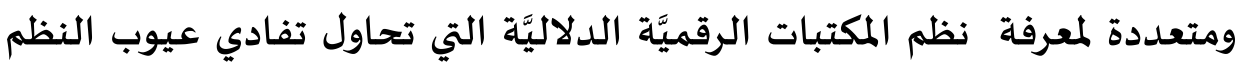
السـابقة، مع توافر طرق جديدة للبحث والاسترجاع، ماهيتها، ومكوناتها،وطرق استرجاعها، وخدماتها، والنظم المتَّبَعَة فيها. 


\section{4}

• التعرُّف على مفردات الإنتاج الفكري الذي تناول موضيوع المكتبات الرقمية الدلالية على اختلاف فئاته وأشكاله ولغته. • التعرُف على سمات وخصائص هذا الرصيد من الإنتاج الفكري: الموضيوعيَّة، والزمنيَّة، واللغويَّة، والشكليَّة، والنوعيَّة. • التعرُّف على اتجاهاته من حيث مدى الاهتمام بالجوانب النظريَّة، والجوانب العلميَّة التطبيقيَّة. • التعرُف على أكثر الدوريات العلميَّة نشرًا للمقالات التي تناولت هذا الموضوع.

• التعرُّف على مدى إسهام الباحثين والمؤلفين في هذا الموضيوع • • الوقوف على مواطن القوة والضعف التي تكثف هذا الرصيد من الإنتاج الفكري. •تقديم اقتراحات للمسـاعدة في تطور سمات وخصائص الإنتاج الفكري العربي في هذا الموضوع.

$$
5
$$

تناولت المراجعة العلميَّة الإنتاج الفكري في العنكبوتية الدلالية بصفة عامة، التهاته

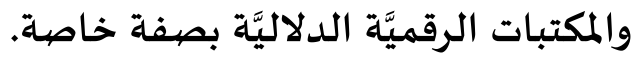

ب- الحدود اللغويَّة:

اقتصرت المراجعة العلميَّة في تغطيتها لمفردات الإنتاج الفكري في العنكبوتية

الدلالية المنشور باللغة العربيَّة والإنجليزية.

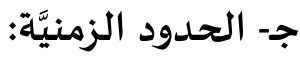
غطَّت المراجعة العلميَّة الإنتاج الفكري الصيادر بين عامي ( 2000 - 2020). 


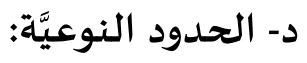

شملت المراجعة العلميَّة الرسائل الجامعيَّة، والمقالات البحثيَّةَ، وأعمال

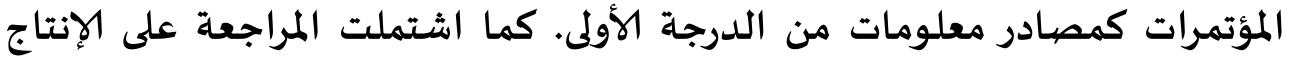
الفكري المنشور في الصيغتين الورقية والإلكترونية.

\section{6}

اعتمد هذه الدراسة على دراسة ببليومترية في وصف وتحليل الإنتاج الفكري في موضوع المكتبات الرقمية الدلالية أنه يعتمد على المنهج الكمي الذي يحول سمات

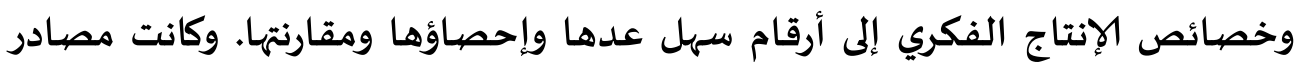
التجميع كالآتي :

مصيادر تجميع الإنتاج الفكري الخاص بموضوع الدراسـة. - دار المنظومة متاح في الرابط التالي:

http://0810g6s38.1103.y.http.search.mandumah.com.mplb.ekb.eg/ MyResearch/Home - اتحاد المكتبات الجامعيَّة المصريَّة والمتاحة على: http://srv1.eulc.edu.eg/eulc_v5/libraries/start.aspx?fn=Login\&Scop $\underline{. e l D=1}$

ملمات، Cybrarians Journal -

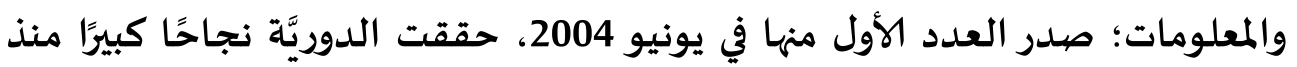

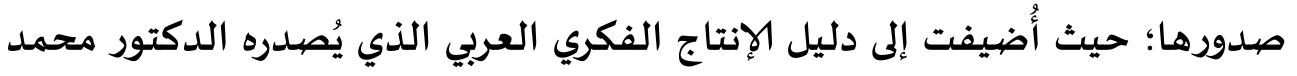

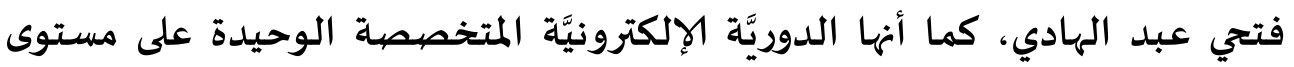

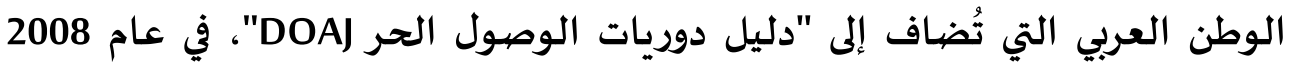

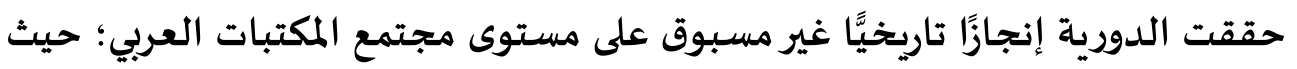

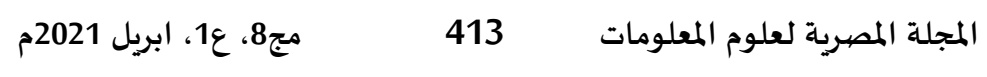


اختيرت الدوريَّة للتكشيف في قواعد البيانات العالميَّة EBSCO لتصبح الدوريَّة العربيَّة الوحيدة من بين الدوريات المتخصصية؛ سواء الإلكترونيَّة أو المطبوعة، لمئه متاحة على:

http://www.cybrarians.info/journal

$$
\text { - قواعد البيانات العالميَّة } 1 \text { ف }
$$

• • البحث في قاعدة Science Direct. • البحث في قاعدة EBSCO host. • البحث في قاعدة Emerald Full-Text. - الباحث العلمي لجوجل Google scholar متاح على: https://scholar.google.com.eg/scholar

https://www.google.com.eg متاح على الرابط التالي: Google جوجل تمَّ البحث باستخدام هذه المصطلحات البحثيَّة فيما يخصُّ اللغة العربيَّة:

$$
\text { المكتبات الرقميَّة الدلاليَّة. }
$$

أما بالنسبة الدراسـات الأجنبيَّة فقد تمَّ البحث بالمصطلحات الآتية:

\section{Semantic digital library}

.Semantic digital libraries

تمَّ استخدام مصطلحات بحثيَّة، وذلك باتباع استراتيجيَّة البحث بالروابط المنطقيَّة (And-Not-Or)؛ وذلك من خلال الكلمات البحثيَّة الآتية:

Semantic Web AND digital library Systems .Semantic Web OR digital library Systems 
تمَّ البحث في بعض القواعد العالميَّة باستخدام الكلمات المفتاحية، وكانت

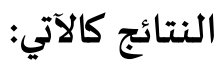
إحصائيات نتائج لبعض القواعد العالمية)
(جدول رقم (1)

\begin{tabular}{|c|c|c|c|c|}
\hline \multicolumn{2}{|c|}{ Semantic digital libraries } & \multicolumn{2}{|c|}{ Semantic digital library } & اسم القاعدة \\
\hline نتائج خاصة & مجمل النتائج & نتائج خاصـة & مجمل النتائج & نتائج \\
\hline 8 & 8.014 & 8 & 8.014 & Science Direct \\
\hline 7 & 822.886 & 5 & 822.842 & EBSCO host \\
\hline 4 & 1674 & 3 & 1673 & Sage \\
\hline 4 & 37,925 & 4 & 37,925 & Proquest \\
\hline 23 & 2.542 .825 & 20 & 2.541 .781 & المجموع \\
\hline
\end{tabular}

وبعد إجراء عملية البحث باستخدام الكلمات المفتاحية ( Semantic digital EBSCO تبين تفوق قاعدة بيانات Semantic digital libraries - library

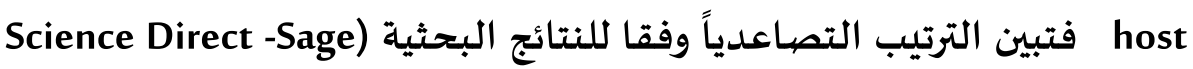
. (Proquest t-EBSCO hos -

\section{• دليل دوريات الوصيول الحر DAOD:}

تمَّ البحث بمصطلحات (Semantic Web AND digital library Systems) أظفرت النتائج عن 15 مقالة بحثيَّة في دليل دوريات الوصول الحر، كما أسفر البحث

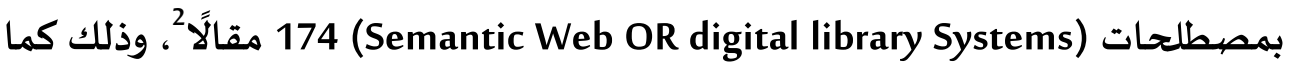
هو واضح من جدول 2. 
جدول رقم (2) نتائج بحث في دليل دوريات الوصول الحرJOO

\begin{tabular}{|c|c|c|}
\hline $\begin{array}{c}\text { Semantic Web OR digital } \\
\text { library Systems }\end{array}$ & $\begin{array}{l}\text { Semantic Web AND } \\
\text { digital library Systems }\end{array}$ & المجال \\
\hline 62 & 9 & ببلوجرافيا وعلم المكتبات \\
\hline 29 & 2 & العلوم الاجتماعيَّة \\
\hline- & 2 & إدارة نظم المعلومات \\
\hline 45 & 1 & طب \\
\hline 27 & 2 & علم المكتبات والمعلومات \\
\hline 13 & 1 & تكنولوجيا \\
\hline 12 & - & تكنولوجيا المعلومات \\
\hline 10 & 1 & تكنولوجيا والهندسة \\
\hline 8 & - & علوم الحاسب الآلي \\
\hline- & 2 & 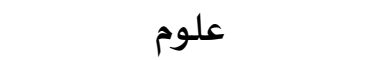 \\
\hline
\end{tabular}

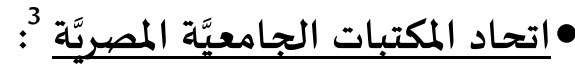

وبالبحث في موقع اتحاد المكتبات الجامعيَّة كانت النتائج كالآتي:

(جدول رقم (3) لنتائج اتحاد المكتبات الجامعية المصرية)

\begin{tabular}{|c|c|c|c|c|}
\hline 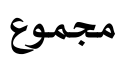 & دوريات & رسائل & 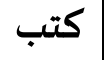 & الكلمات المفتاحيَّة \\
\hline 3 & - & 2 & 1 & Semantic digital library \\
\hline 6 & - & 4 & 2 & Semantic digital libraries \\
\hline $\mathbf{0}$ & - & - & - & Semantic Web AND digital library Systems \\
\hline $\mathbf{0}$ & - & - & - & Semantic Web Or digital library Systems \\
\hline
\end{tabular}


أظفرت النتائج البحثيَّة في قاعدة بيانات دار المنظومة عن خمس دراسـات: ثلاثة بحوث مؤتمرات، ورسالتين جامعيتين، وكتاب واحد فقط؛ أما عن بحوث

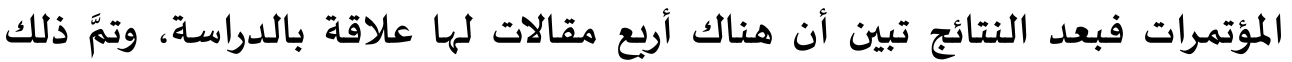
باستخدام الكلمة المفتاحية (الويب الدلالي)، بينما عند البحث بالكلمات بلمات المفتاحية

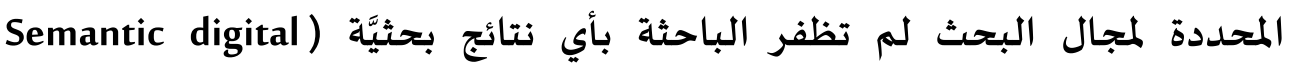

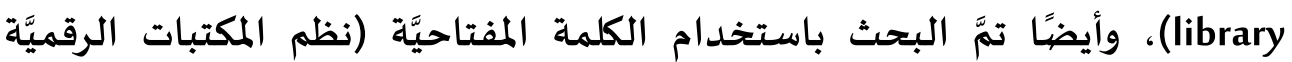
الدلاليَّة).

$$
\text { جدول4 : نتائج البحث في دار المنظومة }
$$

\begin{tabular}{|c|c|c|c|c|c|}
\hline مجموع & مؤتمرات بحوث & 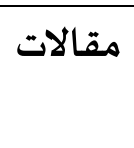 & جامعيَّة & كتب & الكلمة المفتاحية \\
\hline 44 & 3 & 26 & 15 & 2 & الويب الدلالي \\
\hline 0 & - & - & - & - & Semantic digital library \\
\hline $\mathbf{0}$ & - & - & - & - & Semantic digital libraries \\
\hline
\end{tabular}

تمَّ اختيار محرك البحث جوجل لإتمام العملية البحثيَّة، وبعد استخدام الكلمات المفتاحية لكل منه، ومن الباحث العلمي لجوجل كانت النتيجة البحثية كالآتي:

(جدول 5 لعرض النتائج البحثية لكل من Google, Google Scholar)

\begin{tabular}{|c|c|c|}
\hline Google Scholar & Google & الكلمة المفتاحية \\
\hline$\wedge 0 \varepsilon, \ldots$ & $23,900,000$ & Semantic digital library \\
\hline$r q ., \ldots$ & $7,440,000$ & Semantic digital libraries \\
\hline$\neg \varepsilon \wedge, \ldots$ & $21,600,000$ & Semantic Web AND digital library Systems \\
\hline $1, \wedge \varepsilon \cdot, \ldots$ & $65,800,000$ & Semantic Web OR digital library Systems \\
\hline
\end{tabular}


ونستعرض فيما يلي بعضًا من الدراسـات المسترجعة والتي ترتبط بهذه الدراسة الحاليَّة، وقد تمَّ تصنيف هذه الدراسات زمنيَّا وموضوعيَّا؛ حيث تمَّ تقسيمها

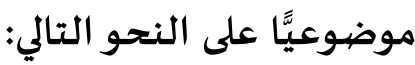

\section{- أوكًا الدراسات التي تناولت ملى تأثير الدلاليَّة على المكتبات الرقميَّة:}

وفيها تم عرض الدراسـات التي تناولت موضوع مدى تأثير الدلاليَّة على المكتبات

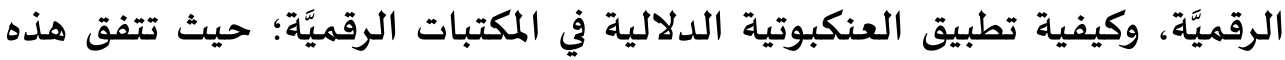
الدراسات مع الدراسـة الحالية في محاولة التعرف على مراحل دخول الدلاليَّة في البيئة الرقميَّة وكيفية التأثر بها، ولكنها تختلف معها في أن الدراسة الحالية تطرقت مراتيها

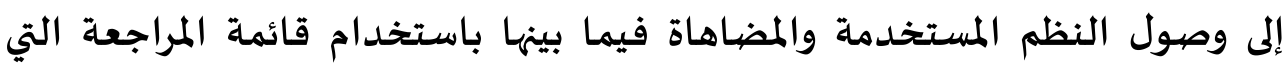
تضهن بعض المعايير للوصول إلى أفضل النظم المستخدمة.

وقد هدفت دراسـة "Kim"7 (2005) إلى تعريف المكتبات الرقميَّة الدلاليَّة كمكتبات رقميَّة يمكنها اكتشاف المعلومات الخفية والمفيدة من كميات هائلة من المن البيانات المخزَّنة باستخدام تقنيات التنقيب عن البيانات؛ مثل: تجميع وتصنيف وتكوين قواعد التنقيب وتقنيات العرض، وتقترح الدراسة لبناء مكتبة رقميَّة دلاليَّة

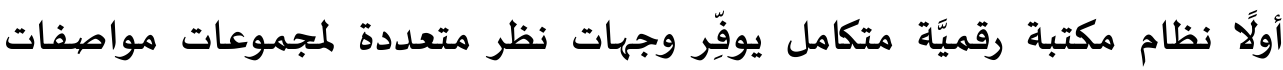
البيانات المتوفرة عن طريق دمج تقنيات البحث والتنقيب عن البيانات، ويوفر هذا النظام ثلاث قيم مضافة؛ هي:

1. توفر خدمة البحث عبر الأرشيف وجهة نظر مصطلح مواصفات البيانات

المجمََّعة.

2. توفر خدمة تصفُّح المفاهيم وجهة نظر موضيوعيَّة لواصفات البيانات المجمَّعـة.

$$
\text { المجلة المصرية لعلوم المعلومات } \quad 418 \quad \text { مج8، ع1، ابريل 2021م }
$$


3. توفِر خدمة ملخص المجموعة وجهة نظر المجموعة لكل مجموعة من

$$
\text { مواصفات البيانات. }
$$

كما تقترح الدراسـة طريقة للتنقيب عن البيانات النصيَّة باستخدام خوارزميَّة

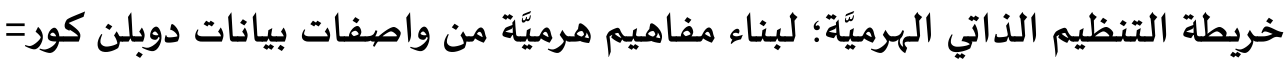

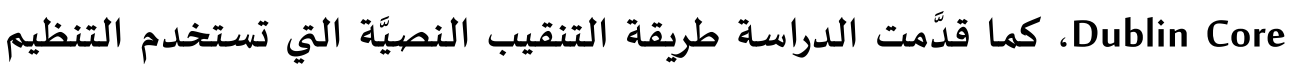

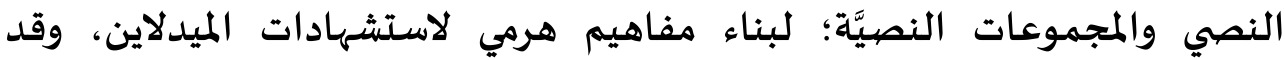

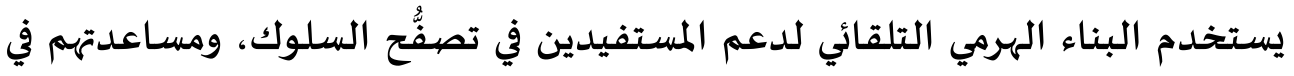

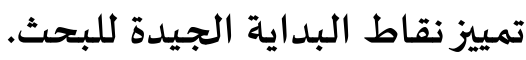

أمَّا عن دراسة "Papadakis" 8 (2005) فقد هدفت إلى سـدِ الفجوة بين شبكة الحاسبات، والاستخدام الدلالي للمعلومات اللذان يجتمعان في بناء المكتبة الرقميَّة،

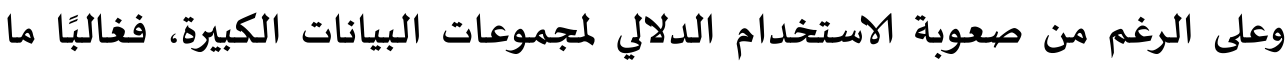

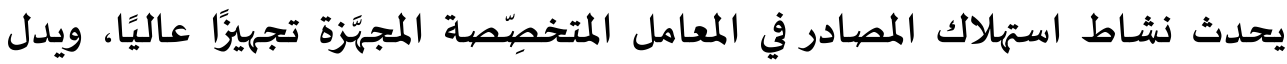

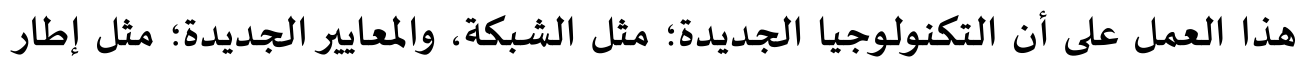

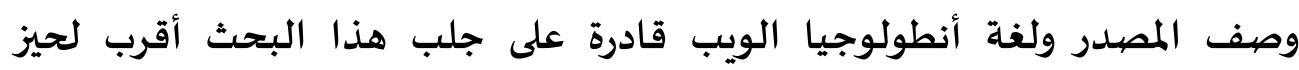

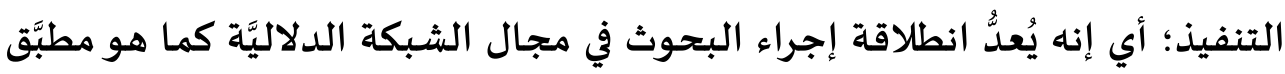
على البنى التحتيَّة للمكتبة الرقميَّة.

بينما تناولت دراسة "Warren" (2005) وصف كيفية استخدام تكنولوجيا

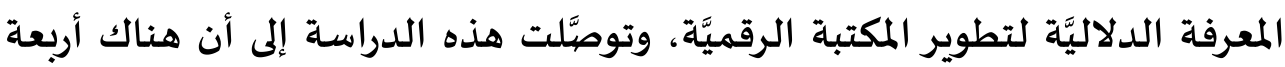

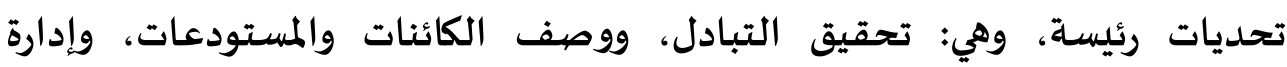

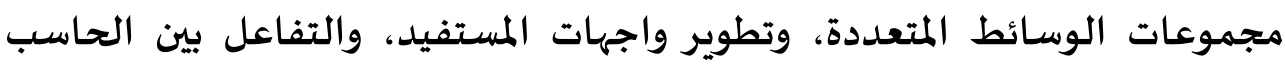
والعنصر البشري .

ومن بين الدراسات أيضًا دراسة "Sicilia" (2005) والتي تهدف إلى تقديم القضية الخاصة بالويب الدلاليَّة؛ وذلك من خلال إجراء أبحاث مكثَّفة في جميع 
أنحاء العالم في المراكز البحثيَّة، كما تمَّت إنجازات نحو الهدف النهائي؛ وهو التعبير،

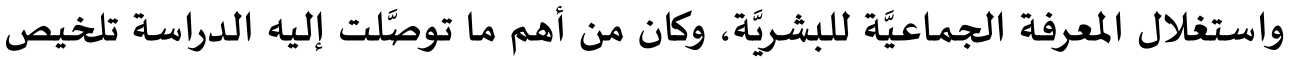

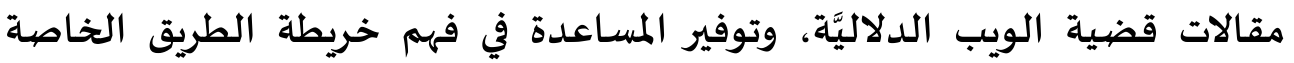

$$
\text { بالويب الدلاليَّة للمكتبات الرقميَّة.10. }
$$

وأمَّا عن الدراسـات العربيَّة؛ فتأتي دراسـة رجب عبد الحميد(2007) في

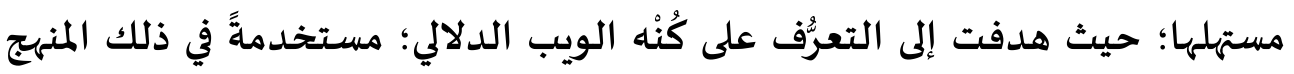
الوصفي التحليلي؛ لبيان أهمية الويب الدلالي، وكيف يمكن للويب الدلالي المساعدة

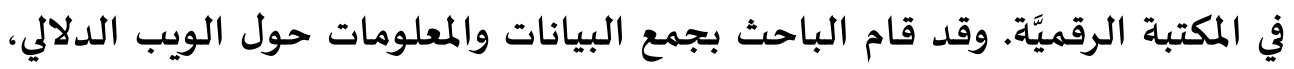

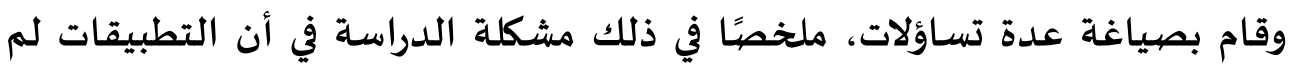

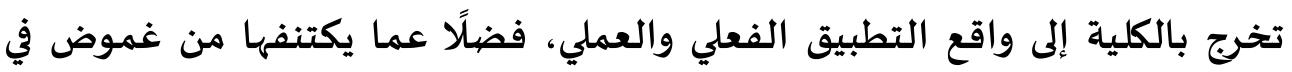

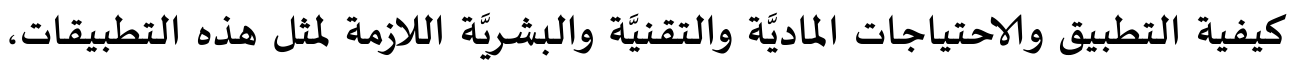
ومدى ملائمة مثل هذه التطبيقات للتطبيق الفعلي في البيئة العربيَّة، وقيام مكتبات

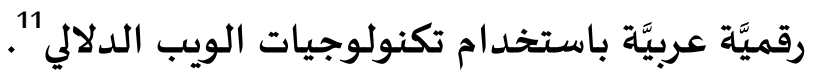

بينما دراسة "Burke " (2009) هدفت إلى مناقشة التعريفات البديلة للويب الدلاليَّة والمسارات الخاصية بها، كما تهدف إلهاه إلى توضيح العلاقة بين الويب الدلاليَّة

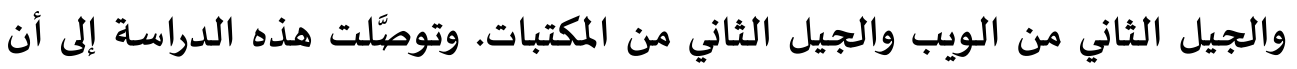

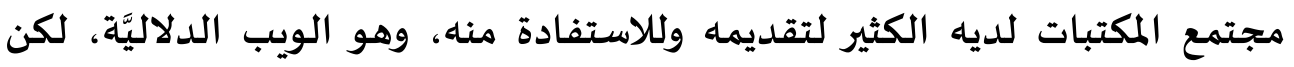

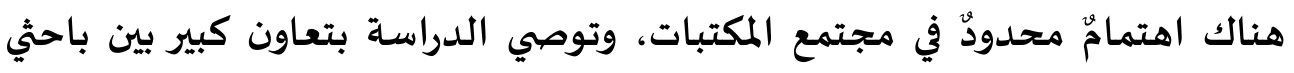

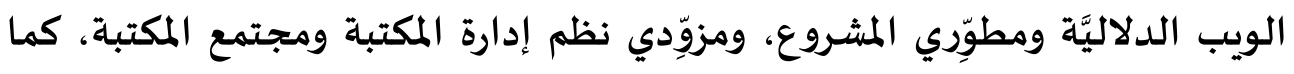

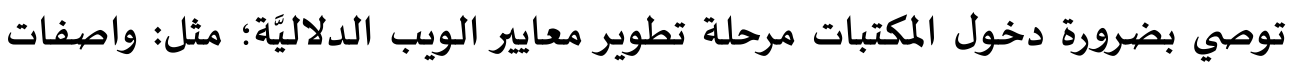
البيانات والتكسونومي توخئ

أما الدراسة الثانية فهي دراسة حسام العباسي (2009) والتي انصلت على

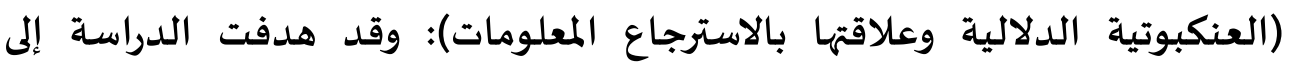

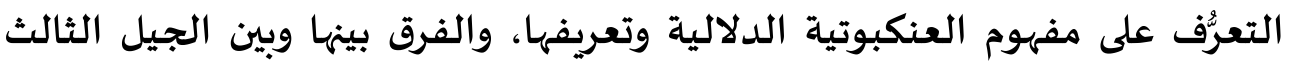

$$
\text { المجلة المصرية لعلوم المعلومات } \quad 420 \quad \text { مج8، ع1، ابريل 2021م }
$$


للعنكبوتية Web 3.0 وأهمية العنكبوتية الدلالية واستخداماتها وتقنياتها، ومحاولة

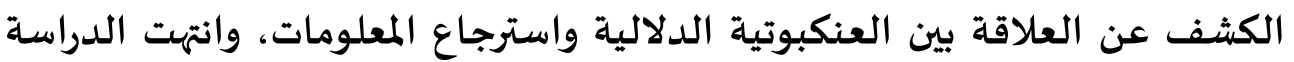

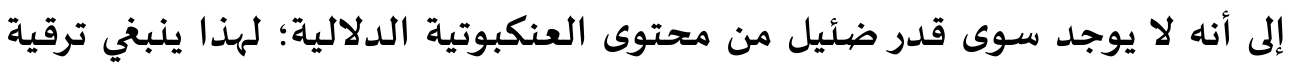

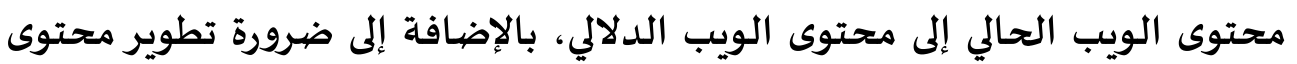

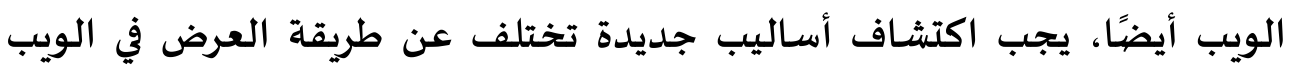

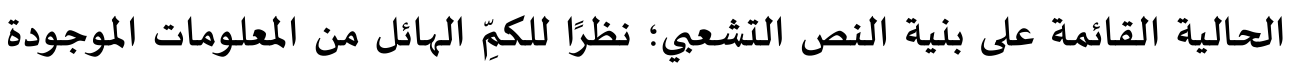

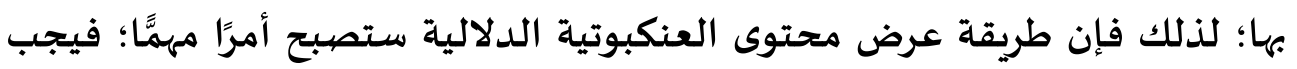

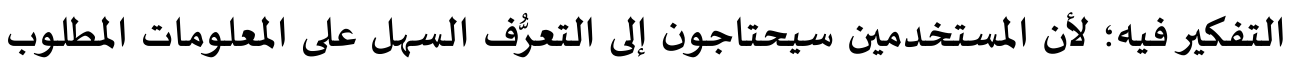
استرجاعها 13

والدراسة الثالثة دراسة غسان مراد وعماد بشير (2010) عن (الكساليب

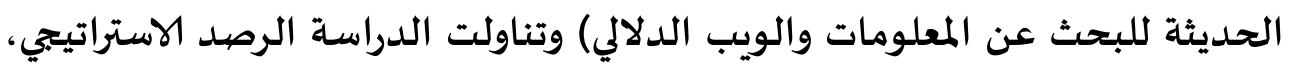

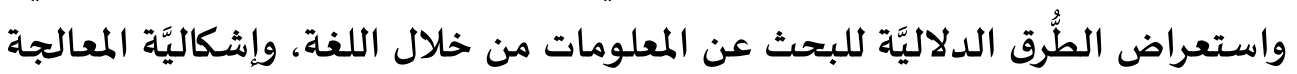

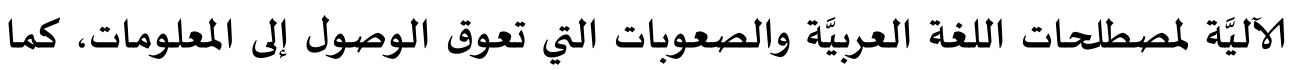

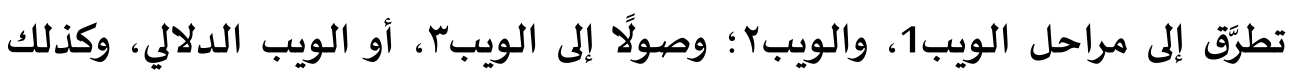

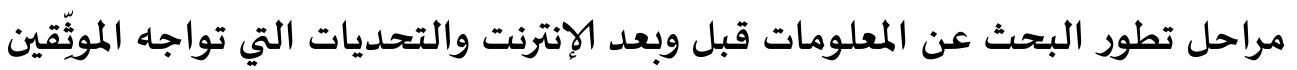

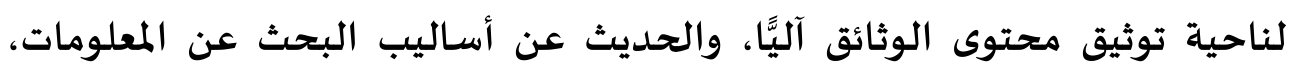

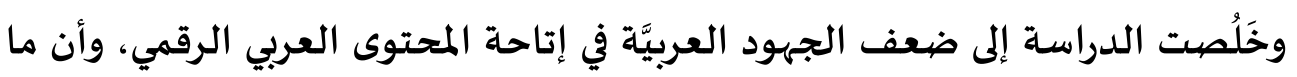

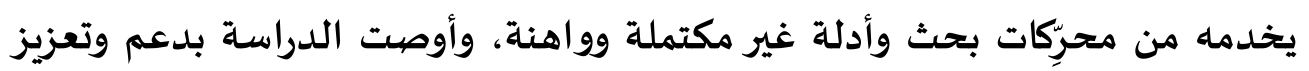

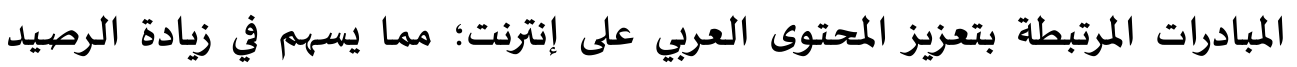
المعرفي

الدراسة الرابعة دراسة نورة الهزاني (2011) تطرَّقت هذه الدراسة إلى شرح

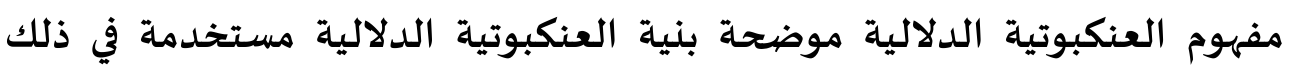

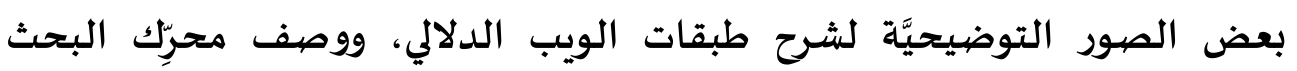

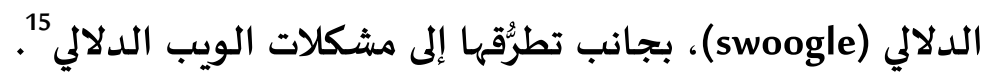


الدراسة الخامسة دراسة أجراها مؤمن النشرتي (2011)عن الشبكة العنكبوتية الدلاليَّة وهدف من خلالها إلى تشخيص وضيع البحث في الشبكاهيكة

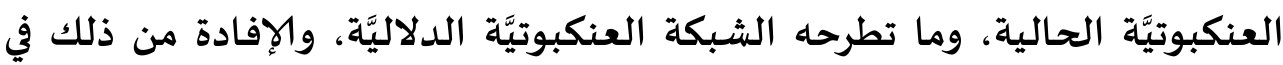

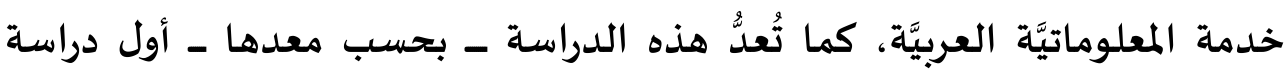

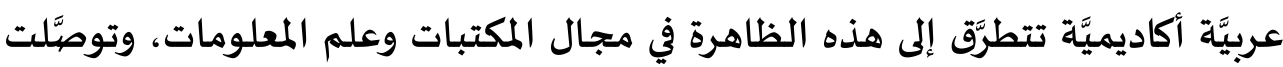

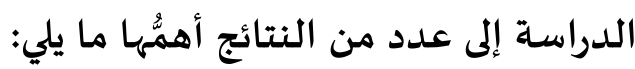

عجز البنية الحالية للشبكة العنكبوتيَّة عن الوفاء بدلالات ما تحويه من

$$
\text { بيانات ومعلومات. }
$$

بيان أوجه القصور التي تعتلي البنية التكويديَّة لمصادر المعلومات المتاحة على الشبكة العنكبوتيَّة؛ من حيث لغة النص الفائق التشعبي (HTML)

$$
\text { التي تقف عقبة في توفير دلالات المحتوى. }
$$

وقد أوصت الدراسة بعدد من التوصيات منها: ضرورة العمل على التخلي عن

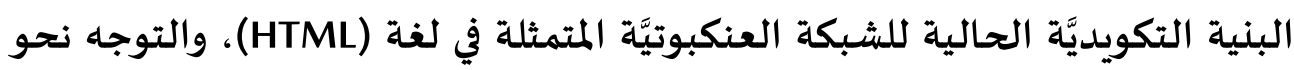
الاعتماد على التكويد بلغة XML، وكذلك إثراء المحتوى العربي بالأنطولوجيات العامة العبه والمتخصصية.16.

الدراسة السـادسة دراسة مؤمن السيد النشرتي (2012) عن محرِكات البحث

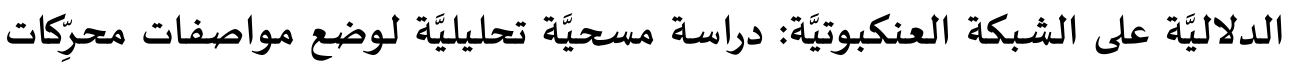

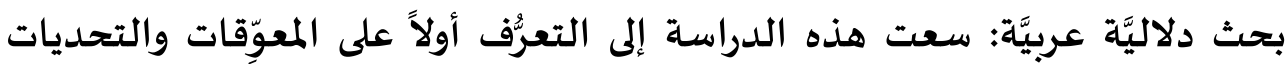

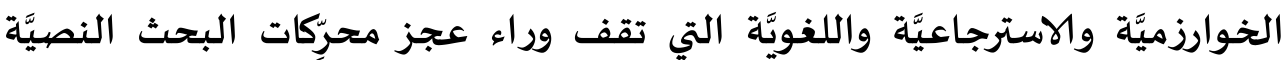
التقليديَّة عن تلبية احتياجات المستفيدين من المطابقة والمضياهاة بين الاحتياجات

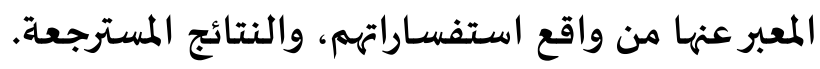

كما تطرَّقت الدراسة إلى محرِكات البحث الدلاليَّة كفئة جديدة من أدوات

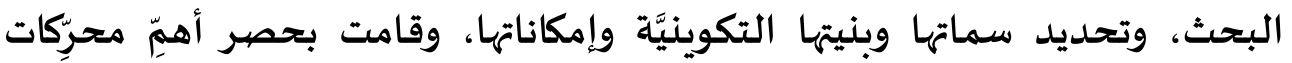

$$
\text { المجلة المصبرية لعلوم المعلومات } \quad 422 \quad \quad \quad \quad \quad \quad \quad
$$


البحث الدلاليَّة على الويب، ودراسـة بنيتها المعماريَّة، وعملت الدراسة على إنشـاء وثيقة؛ بناءً على مواصفات العنكبوتية الدلاليةباللغة العربيَّة، والتأكد من صهاحيتها

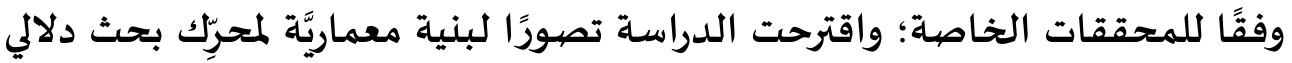
عربي 17

الدراسة السابعة دراسـة الأكلبي (2012) عن تطبيقات الويب الدلالي في بيئة المعرفة اتَّبع فيها الباحث المنهج الوصفي التحليلي الذي يتلاءم مع هذا النوع من

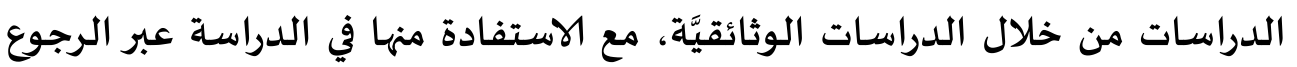

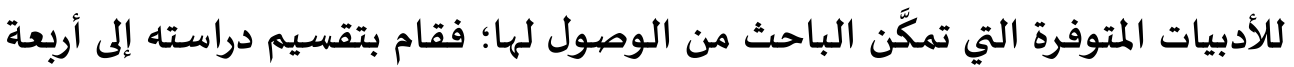

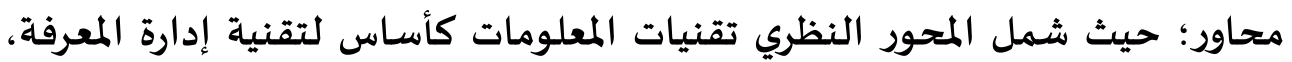
وقام بعرض أنواعها كالآتي: - ماتئ

(شبكة الإنترنت- البريد الإلكتروني- الفهارس الآلية للمكتبات- أقراص الليزر

$$
\text { المتراصية - محرِكات البحث). }
$$

ومنها تطرَّق للتوصل إلى تعريف للويب الدلالي، وذلك بعد سرد بعض المفاهيم

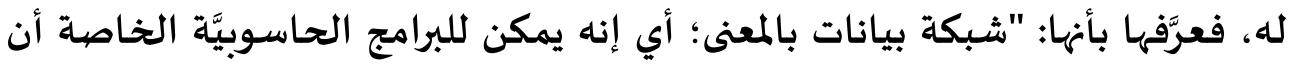

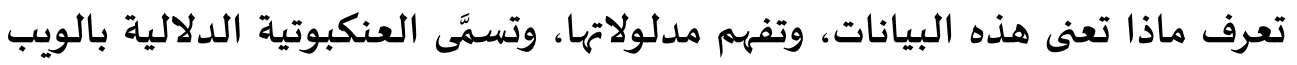
ذي الدلالة اللفظيَّة، أو ويب دلالات المعاني.

أمَّا عن المحور الثاني فهو المحور التقني: ويتمثل في العنكبوتية الدلالية

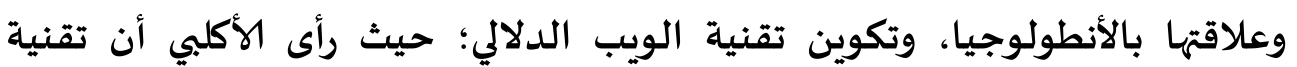
الويب تتألَّف من: بالانطولئ

- لغة لتنسيق تبادل البيانات: مثل لغة إطار وصف المصدر (RDF)، أو بدائلها مثل: (RDF/XML ). 
- مخططات العلاقات: مثل:(RDF Schema) ، ولغة وجودية الويب(OWL) ، تُسهيّل عملية توصيف المفاهيم والمصططحات والعلاقات ضهمن مجال معين. - محرِّك الاستدلال: والذي يحتوي على قواعد استدلاليَّة تستخدم اللغتين السابق ذكرهما، ولغات أخرى مبنية عليها لإعطاء نتائج منطقيَّة تمامًا كما يفكر لوسريك البشر.

أمَّا عن المحور الثالث فكان تحت عنوان: الأهمية والتوظيف، عرض فيه أهمية الويب الدلالي؛ موضحًا في ذلك استخداماته، وربطه في المحور الرابع بعلاقة كل من العنكبوتية الدلالية والويب 3.0، مستنتجًا في نهاية دراسته بعض الدوهي النتائج التي

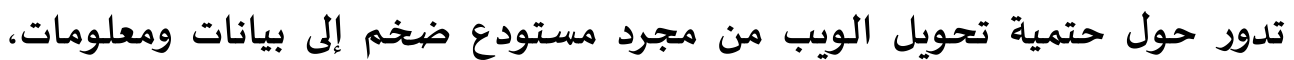
وذلك باستخدام تقنية الويب الدلالي 18 .

الدراسة الثامنة دراسة نرمين إبراهيم (2014) تطرَّقت هذه الدراسة إلى

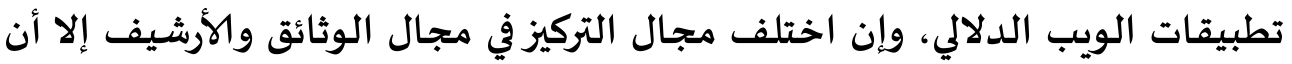

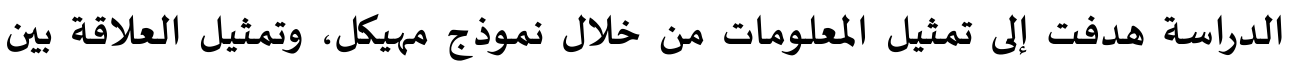

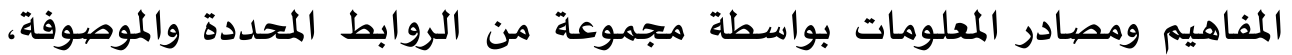
بالإضافة إلى التعرُف على صيغ العنكبوتية الدلالية وتقنياته عبر الأجيال الويب. وتضمَّنت فصول الدراسـة إدارة الوثائق عبر أجيال الويب وصياغة العنكبوتية

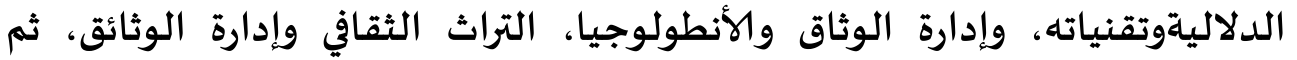
خرائط الموضوعات والوصف الأرشيفي، وأوصت الدراسة بضرورة إثراء المحتوى

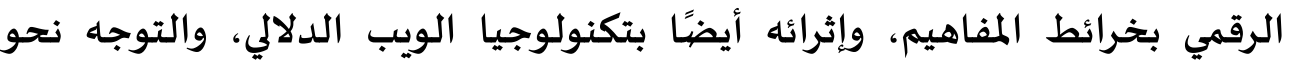
التكويد بلغة XML والتوجه نحو نمذجة المحتوى المتاح على العنكبوتيَّة في مجموعة من نماذج البيانات

الدراسة التاسعة دراسة هبة محمود (2017) تناولت هذه الدراسـة أيضًا واقع تطبيق تكنولوجيا الويب الدلالي، وقد تلخَّصت في النقاش حول تطبيق تقنيات

$$
\text { المجلة المصرية لعلوم المعلومات } \quad 424 \quad \quad \quad \quad \quad \quad
$$


العنكبوتية الدلاليةقفي المكتبة الرقميَّة، والمشاكل التي تواجهها، وأهمية استخدام

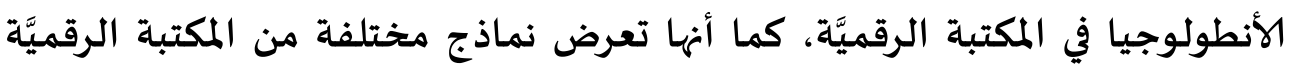
مثل S5 كنموذج رسمي.

وأوضحت الدراسـة أهمية إضفاء الدلاليَّة نحو الرقمنة؛ وذلك لتفادي بعض

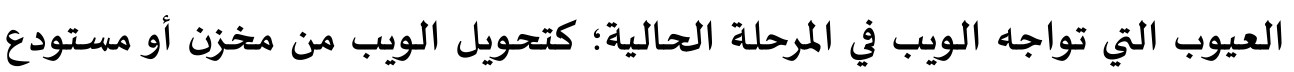

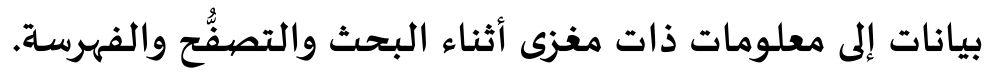

وقد تطرَّقت هذه الدراسة أيضًا إلى استخدام نظام مكتبة رقمية يستخدم تكنولوجيا العنكبوتية الدلالية في تطبيقها؛ وذلك لتقييم البحث، ويتيح هذا النظام

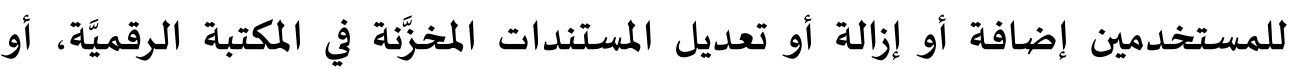

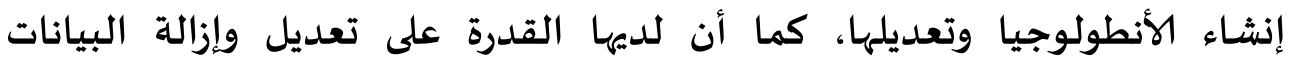

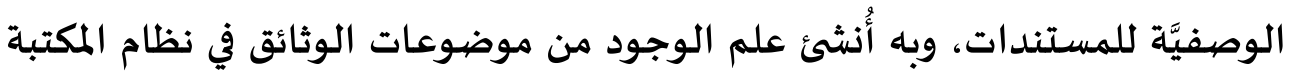

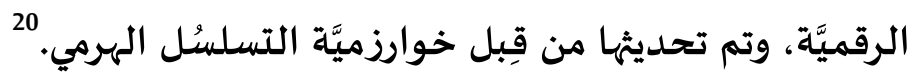

ثانيًا : الدراسات التي تنتاولت الارتباط بين الأنطولوجيا والصنكبوتية الدلالية وتأثيرها على المكتبـات الرقويَّة اللدلاليَّة:

تناولت هذه الدراسـات مدى ارتباط الأنطولوجيا بالعنكبوتية الدلالية ومدى

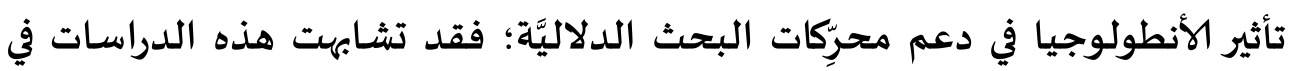
بعض النقاط البحثيَّة كالتعريف بالأنطولوجيا، ومدلولهيا، وليحيا، ومفهومها.

الدراسة الأولى دراسة أحمد بدر (2002) بعنوان الأنطولوجيا وعلاقتها بعلم

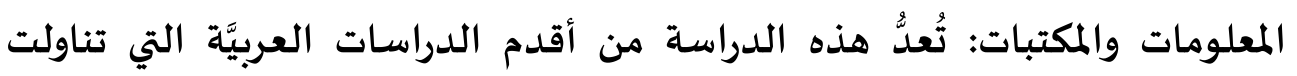

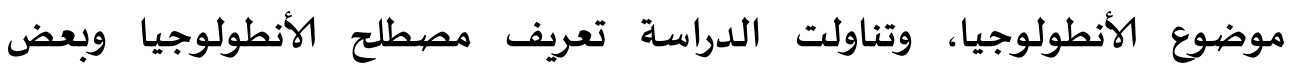

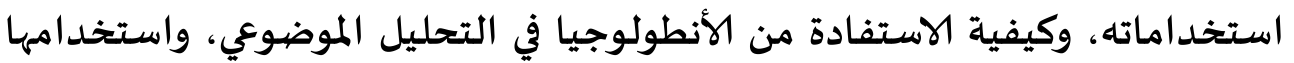

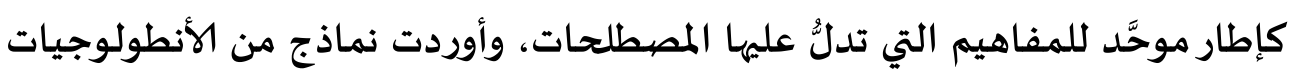


الكاملة وتطبيقاتها، وناقشت مدى إفادة الأنطولوجيا الحديثة من إنتاج علماء

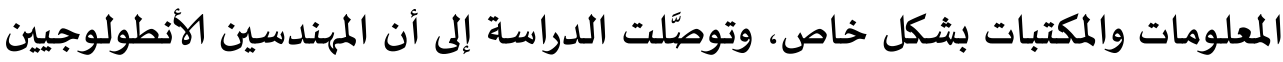

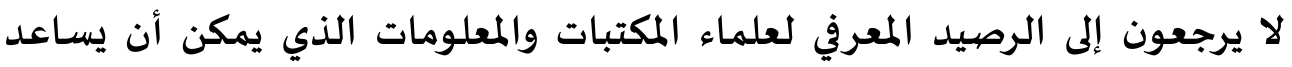

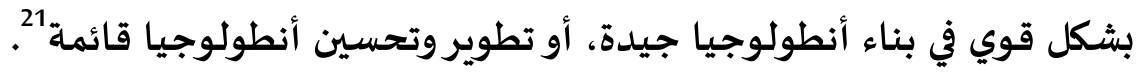

كما قدَّمت دراسة "pampati" (2003) أسلوبًا جديدًا غير مسبق يجمع بين التنقيب عن البيانات واستخدام الأنطولوجيا، ما يتيح عرض بيانات محل البحث في

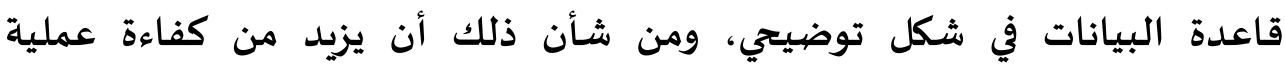

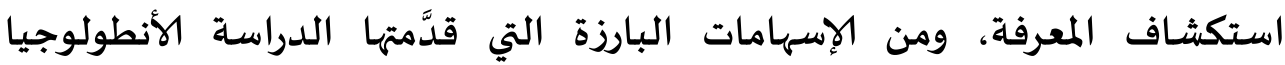
الخارجية، ولتطبيق هذه المفاهيم قامت الباحثة بتصيميم أنطولوجيتين هما

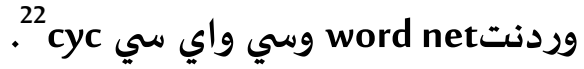

الدراسة الثانية دراسة "LI WEB" (2005) هدفت هذه الدراسة إلى التعرُّف

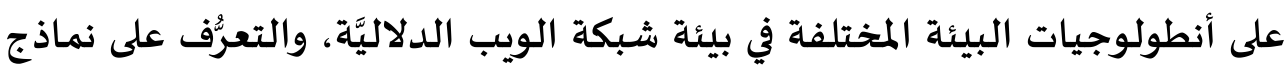

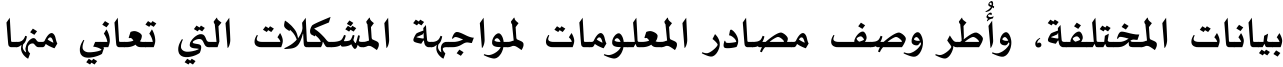

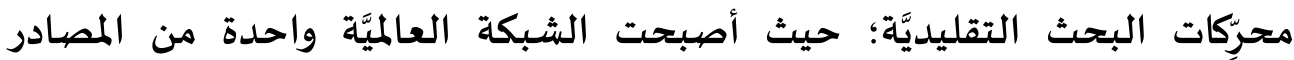

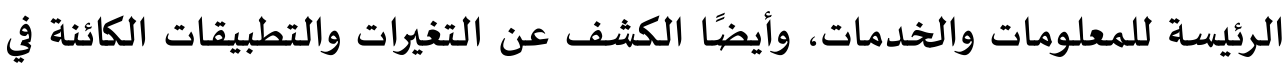
البحث الدلالي؛ من خلال تقديم دلالات البيانات الأساسية، وأدبيات تمكين الاستين الاستدلال الآلي على الأنطولوجيا، ورصد التهيد التيبرات التي تنطوي على البيانات في

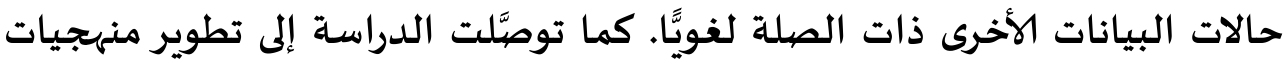

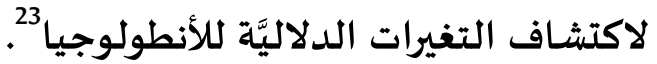

الدراسة الثالثة دراسـة "JRG Pulid" (2006) هدفت هذه الدراسـة إلى تحديد

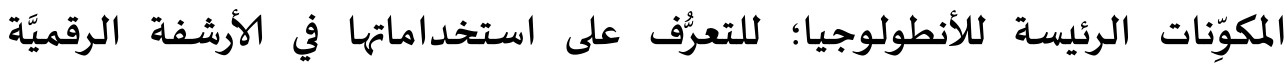

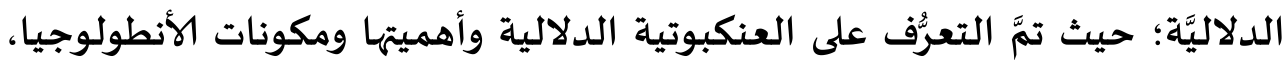
وذلك باستخدام خرائط التنظيم الذاتي (SOM)؛ حيث يمثل نظامًا لتمثيل المعرفة الواردة في مجال معين، وتستخدم في الأرشفة الرقميَّة لتجميع وعرض مكوّيّنات

$$
\text { المجلة المصرية لعلوم المعلومات } \quad 426 \quad \quad \quad \quad \quad \quad
$$


الأنطولوجيا لها، ويدعم آليات استكشاف المجالات، ويسمح لمكوّنات المعرفة أن يتمَّ

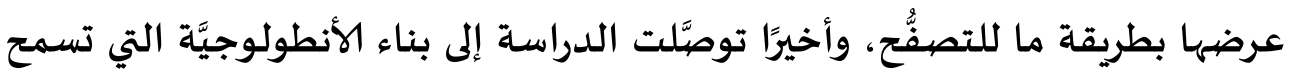
بتطبيق الأنطولوجيات في مجال الأرشفة الرقميَّة. 24

الدراسة الرابعة دراسـة وصال إبراهيم (2010) هدفت هذه الدراسة إلى التعرُّف على مفهوم الأنطولوجيا ونظم المعرفة والمعلومات، ونظم المعرفة والمعلومات ودورهما في تطور المجتمعات وتقليص الفجوة الرقميَّة بين المجتمعات، وردم الهوة الثقافيَّة، كما تبحث الدراسة في مصطلحي الأنطولوجيا ونظم المعرفة، وموقع الدول على خارطة المجتمع المعرفي والمصطلحي والتحديات التي تواجه البلدان النامية في

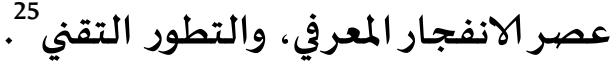

الدراسة الخامسة دراسة مصطفي الجرار (2011) كان من أهمِّ استنتاجاتها،

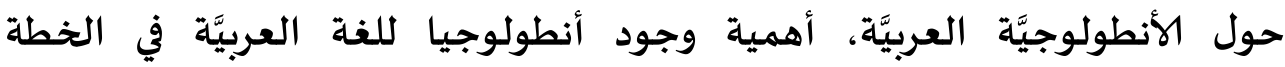
المستقبليَّة؛ وذلك بسبب زيادة التوجه نحو استخدام اللغة العربيَّة في كثير من التطبيقات، ولقد نمت الحاجة مؤخرًا وتعددت المجالات بمَّا يستدعي وجود وسيط يقوم بتعريف المصطلحات العربيَّة بمفاهيم دلاليَّة تتيح المجال لفهرم المشاركة وتبادل البيانات بصورة واضحة ودون أي غموض، وعليه انطلق مشروع بناء أنطولوجيا

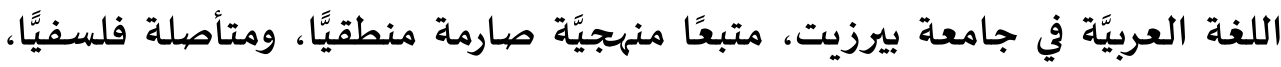

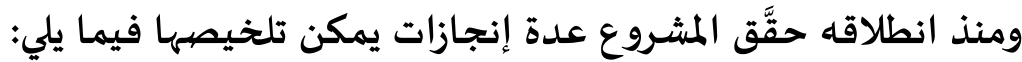

- بناء المستويات العُليا لأنطولوجيا اللغة العربيَّة، والتي تُشكل أسـاس

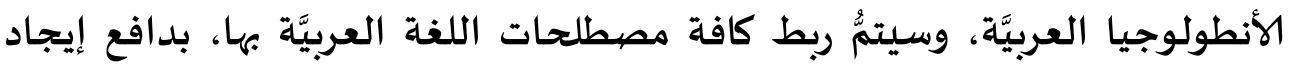
علاقات مفاهيميَّة مؤصَّلة بين المعاني العربيَّة، والتحقق من صحسة هذه العلاقات. - تمَّ إنشاء قاعدة بيانات تحوي ما يُقارب ثلاثين ألف مصطلح عربي ومعانيها المعرفة دلاليَّا، وأنطولوجياء ممَّا يشكل اللبنة الأساسيَّة للأنطولوجياء العابِ العربيَّة. 
- إنشاء برنامج حاسوب مبنى على خوارزميَّة ذكية تعمل على الربط بين

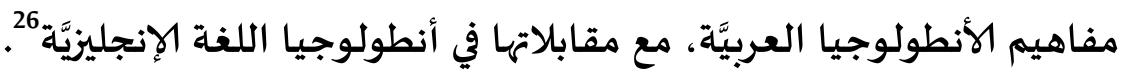

الدراسة السـادسة دراسة ضياء الدين (2012) تناولت هذه الدراسة الأنطولوجيات ونظم استرجاع المعلومات لتوضيح مفهوم الأنطولوجيا مع ذكر أنواعها

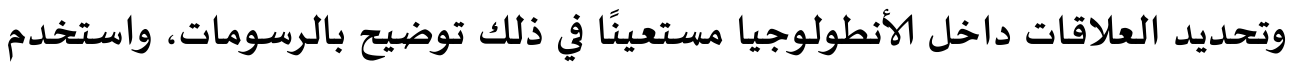
الباحث في دراسته المنهج الوصفي التحليلي، والذي قام بجمع البيانات حول الأنطولوجيا وأنواعها وكيفية بناء الأنطولوجيا، وتحديد العاجية العلاقة الداخليَّة (علاقة

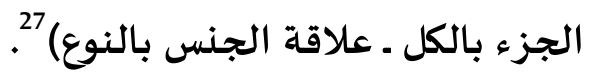

الدراسـة السـابعة دراسة مؤمن النشرتي (2012) عن "نحو التكامل المعرفي من واقع توظيف الأنطولوجيا في إطار التنقيب عن البيانات، دراسة تحليليَّة": هدفت التهاتئي هذه الدراسة إلى التأصيل النظري للأنطولوجيات في إطار التنقيب عن عن التوجيب البيانات،

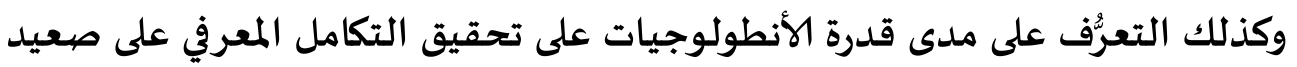

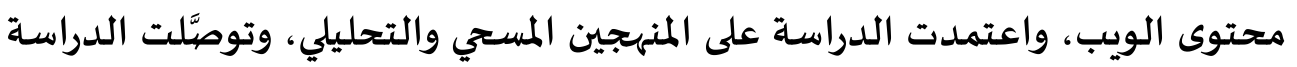

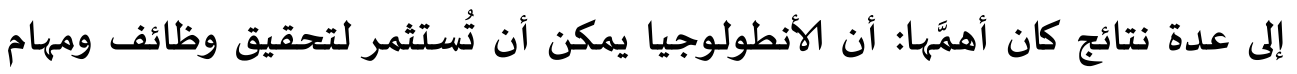

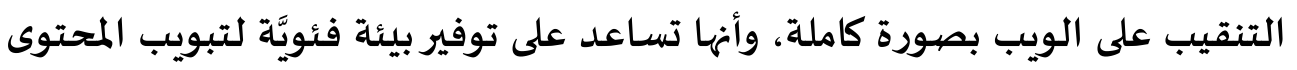

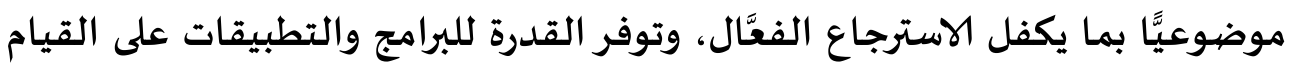
بمهمة إدارة ودمج وتكامل المعرفة.28

وأوصتت الدراسة بتوصيات أهمُّها: ضرورة تطوير محرِكات بحث دلاليَّة عربيَّة

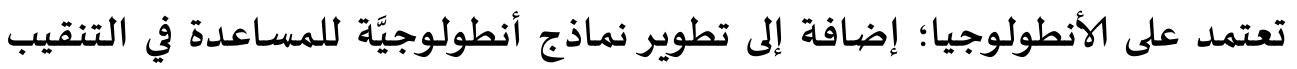

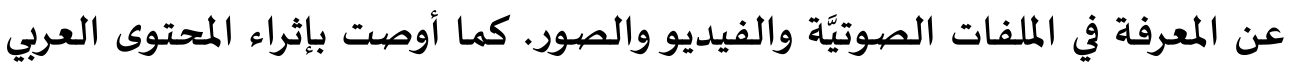
بالأنطولوجيا العامة والمتخصصية، كما تؤكِّد هذه الدراسـة على أهمية قيام دراسـات تطبيقيَّة لبناء أنطولوجيا لونيا عربيَّة. 
الدراسة الثامنة دراسـة مؤمن النشرتي (2012) حول محرِّات البحث الدلاليَّة

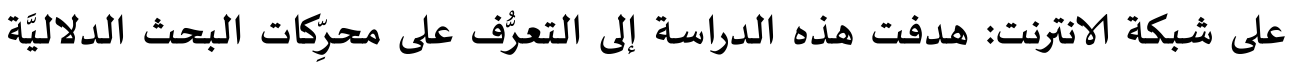

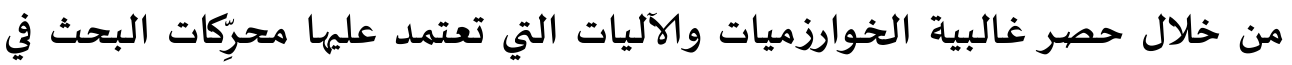
استرجاع المحتوى، وخوارزميات عملها؛ وذلك لدراسة التحديات التي تواجه

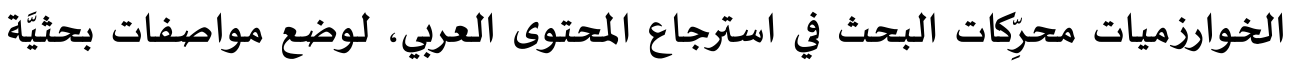
دلاليَّة عربيَّة.

وتمثَّلت نتائج الدراسة في حصر التحديات التي تواجه محرِّات البحث بعد

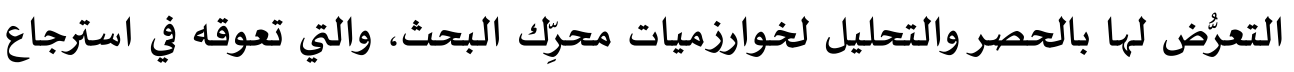

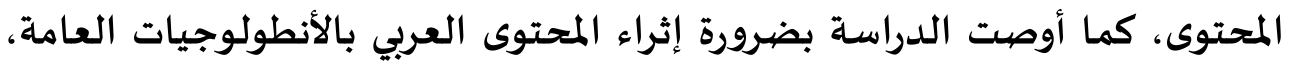

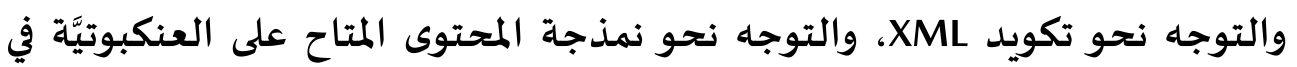
مجموعة من نماذج البيانات Data Model.

وتعتبر دراستنا هذه من هذه الناحية دراسة تكميليَّة؛ حيث تطرَّقت إلى مناقشَة

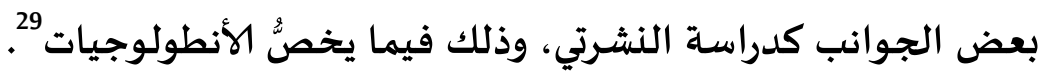
لبناء أنطولوجيا علم المكتبات والمعلومات، وأوصت الدراسة بضرورة الالتزام بمنهجيَّة علميَّة لتصيميم الأنطولوجيات. 30 الدراسة التاسعة دراسة هندي عبد الله (2013) حول بناء أنطولوجيات علم

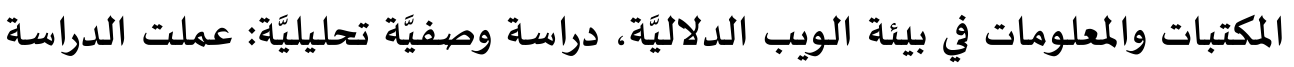

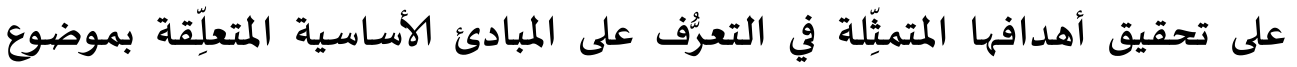

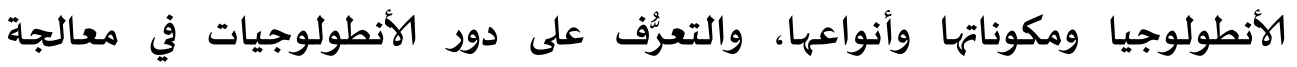

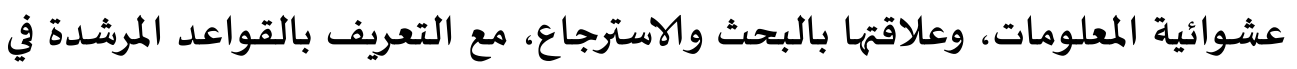

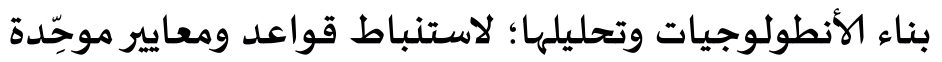

الدراسة العاشرة عبد الناصر سالم (2014) تناولت هذه الدراسة بالوصف والتحليل والتقييم دور محررات الأنطولوجيا مفتوحة المصددر في دعم عمليات إدارة

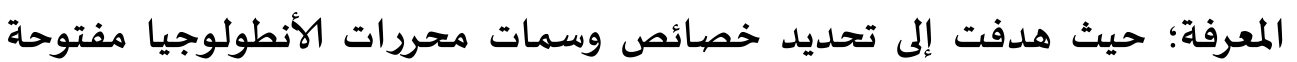


المصددر، والتعرُف على دورها في دعم عمليات إدارة المعرفة، والوقوف على طرق

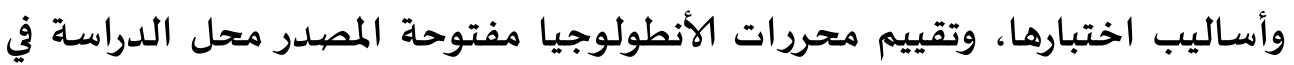

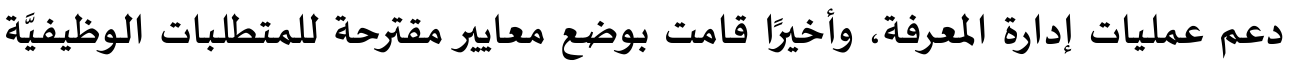
لتطوير وتقييم محررات الأنطولوجيا مفتوحة المصددر في دعم عمليات إدارة المعرفة.

وقد استخدم الباحث المنهج الوصفي الوثائقي في حصر محررات الأنطولوجيا

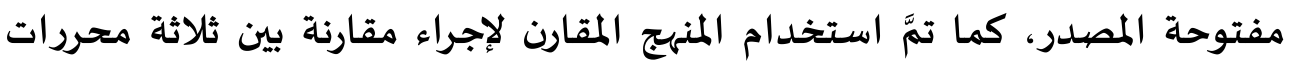

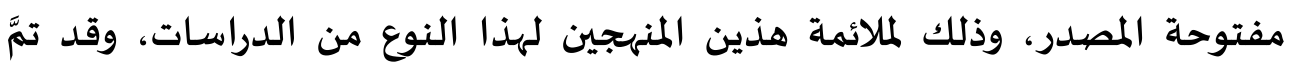

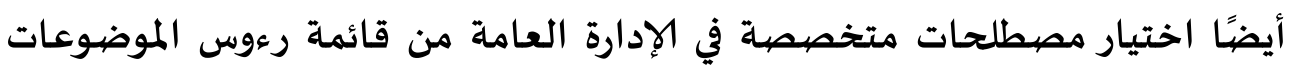

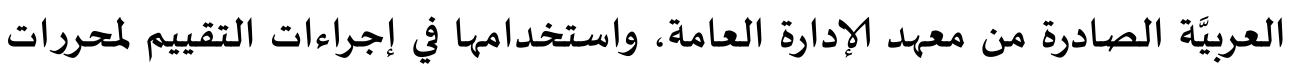

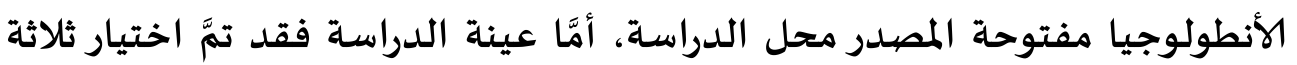
محررات للأنطولوجيا مفتوحة. مفتوجة المهيلور مجتول

الدراسة الحادي عشر دراسـة أحمد فرج أحمد (2015) عن أنطولوجيا

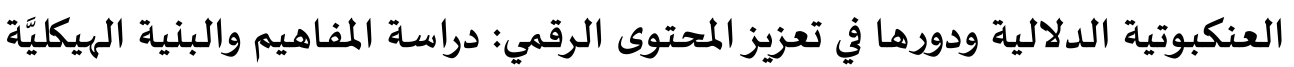

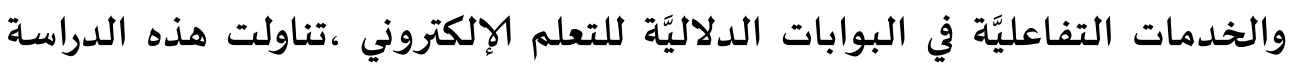

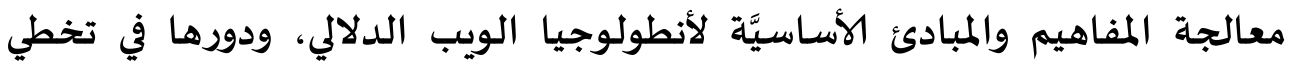

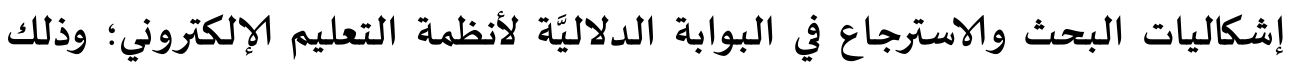

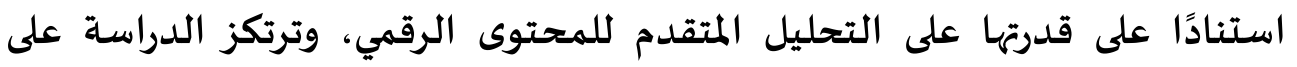

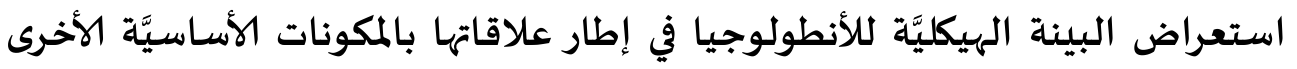
للويب الدلالي؛ وذلك بغرض التعرُّف على دورها، وبيان آثارها في تعزيز الخدماتي التهات

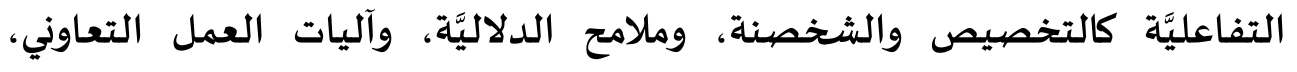

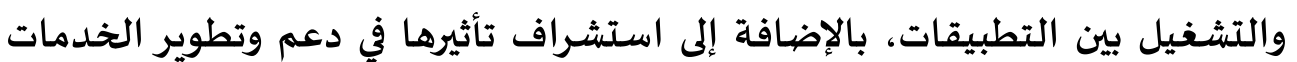

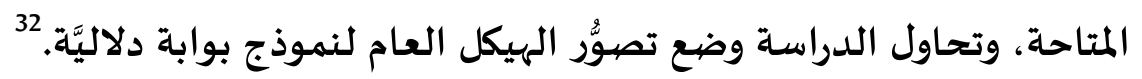


ثالثًا : اللدراسات التي تحدَّثت عن نظم المكتبات الرقميَّة الدلاليَّة :

نستعرض فيما يلي بعضًا من الدراسات التي تحدَّثت عن نظم المكتبات

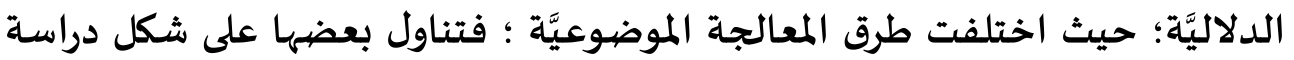
حالة لنظام منفرد، ومنها ما تناول نظم الؤشففة في المكتبات الرقميَّة الدلاليَّة، والآخر تناول بعض النظم بشكل وصفي؛ فتتشابه هذه الدراسات مع الدراسة الحالية في بعض من عينة الدراسـة، ولكن تختلف الدراسة الحالية بطرق تناولها للنظم عينة

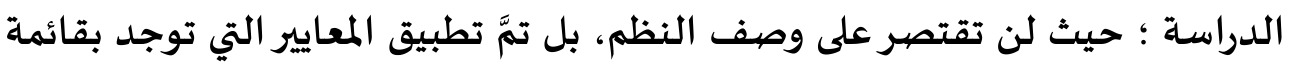

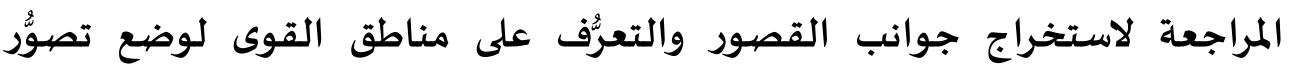
المكتبات الرقميَّة الدلاليَّة في البيئة العربيَّة.

الدراسة الأولى دراسة عواطف على مكاوي (2006) تناولت هذه الدراسة البرمجيات مفتوحة المصيدر من حيث: تعريفها وتاريخ ظهورها، وإيجابياتها وسلبيتها

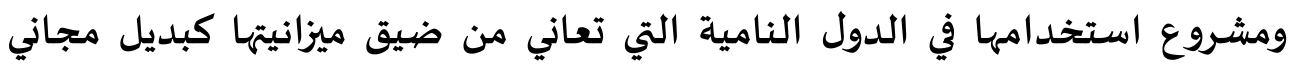

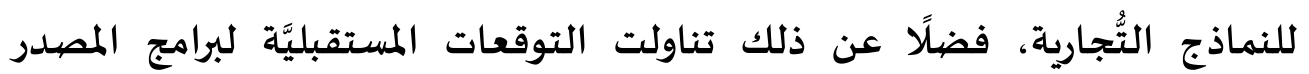
المفتوح، كما استعرضت بعض النماذج المكتبيَّة مفتوحة المصيدر .

الدراسة الثانية: دراسة رحاب فايز (2009) بعنوان نظم المكتبات الرقميَّة

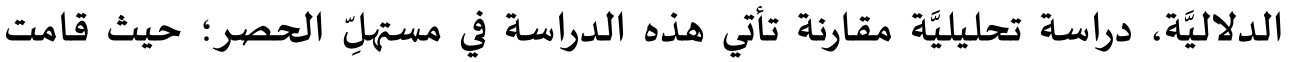

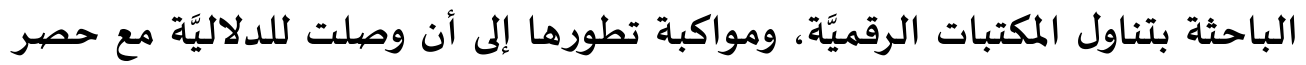

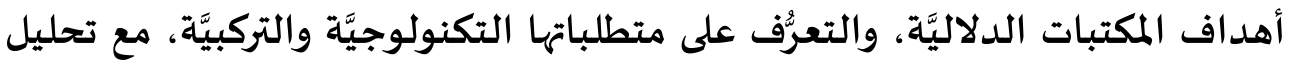
_ لبعض النظم الدلاليَّة (BRICKS_SIMILE _ JeromeDL_ Greenstone _ Fedora)

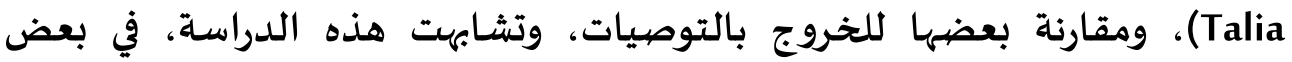

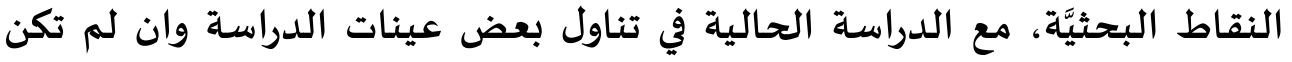

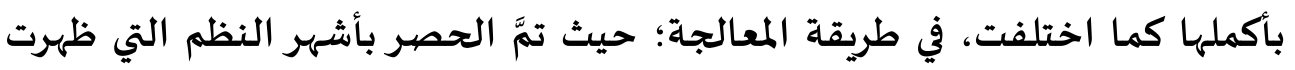

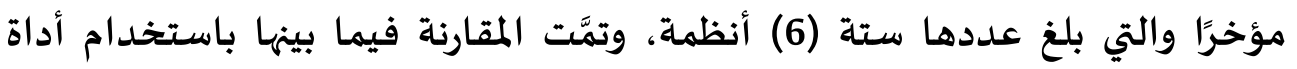
قائمة المراجعة؛ وذلك لوضع تصور للمكتبات الرقميَّة الدلاليَّة في البيئة العربية.34. 
الدراسة الثالثة دراسـة عمرو حسن فتوح (2010) اهتمت الدراسـة بمناقشة موضيوع المكتبات الرقميَّة، وبيان مدى الحاجة إليها في وقتنا الحالي، والنظم مفتوحة

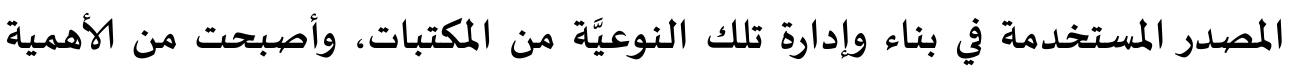

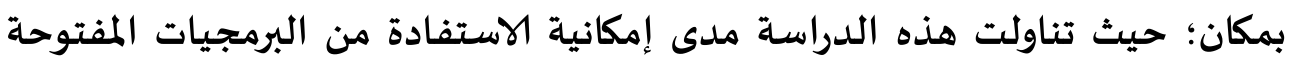

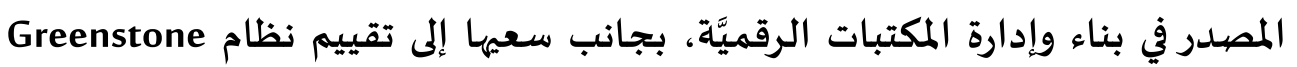

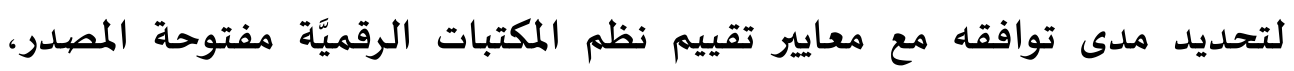

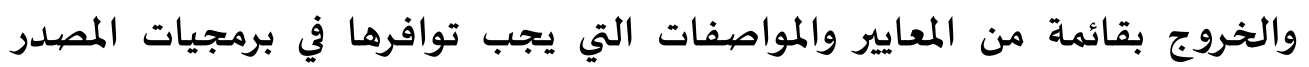

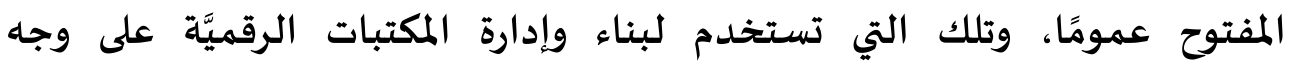
الخصوص، والتي تمَّ الاستعانة ببعضٍ من معاييرها، ووضع تصهور مقترح لمراحل تقييم البرمجيات مفتوحة المصيدر 35

الدراسة الرابعة دراسة سعاد عوض (2013) عن تطبيق تكنولوجيا العنكبوتية الدلالية في المكتبة الرقميَّة والتي تناقش فيها تعريف العنكبوتية الدلالية وعلاقتها

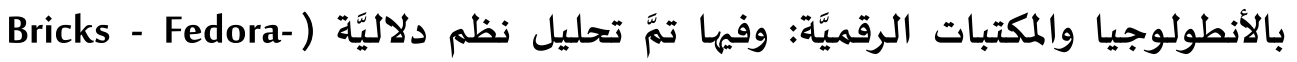

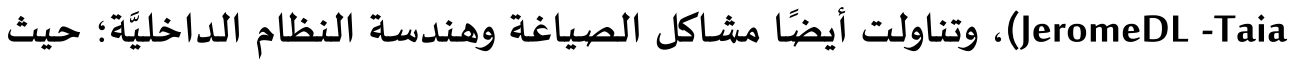

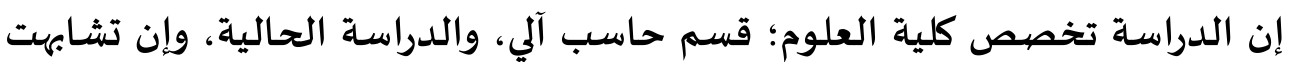

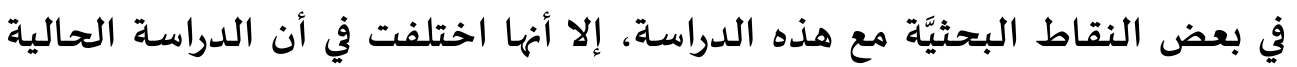

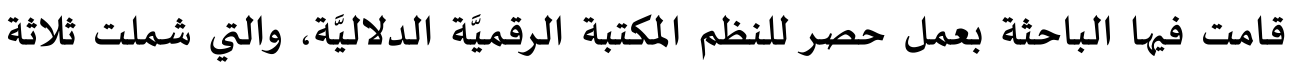
عشر (13) نظامًا، ولم يقتصر على التحليل بل تمَّت المقارنة باستخدام قائمة

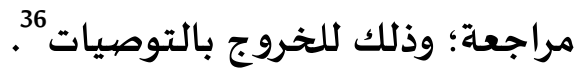

الدراسة الخامسة دراسة أحمد ماهر خفاجة (2014) بعنوان (البرمجيات

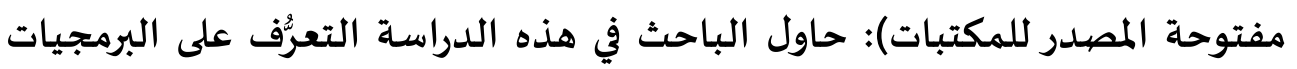

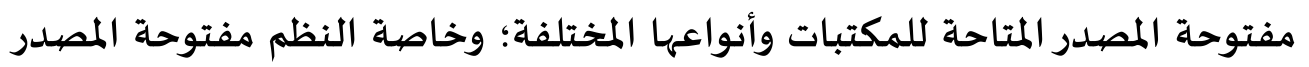

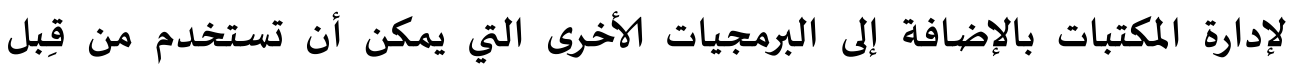

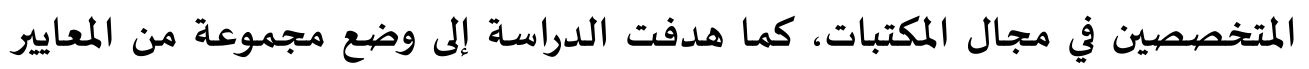

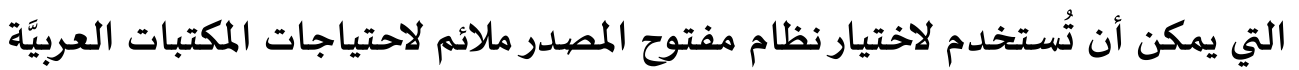

$$
\text { المجلة المصرية لعلوم المعلومات } \quad 432 \quad \quad \quad \quad \quad \quad
$$


من خلال فحص ثلاثة عشر (13) نظامًا آليَّا مفتوح المصبدر لإدارة المكتبات، واعتمدت

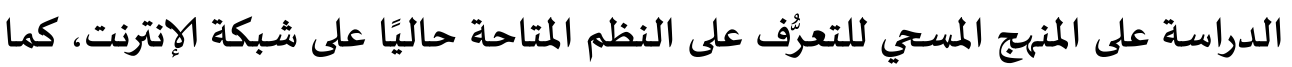

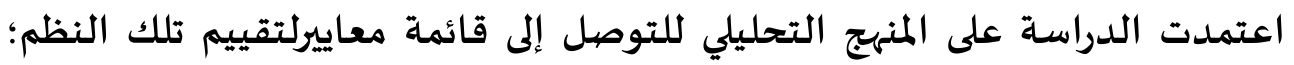

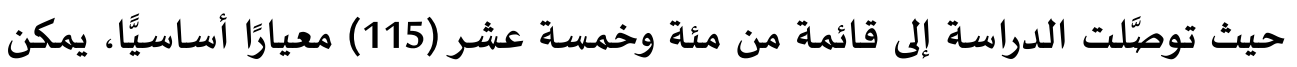
أن يُستخدم إلى جانب المعايير التي طرحتها دراسة (رندا إبراهيم) لتقييم النظم مئه

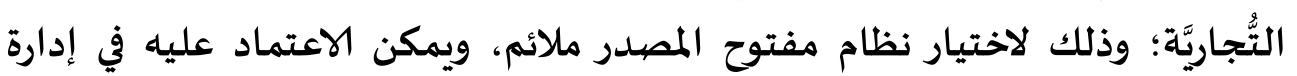

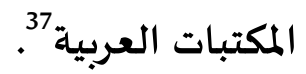

الدراسة السادسة دراسة مرثا نبيل صبري (2016) بعنوان نظم الأرشفة

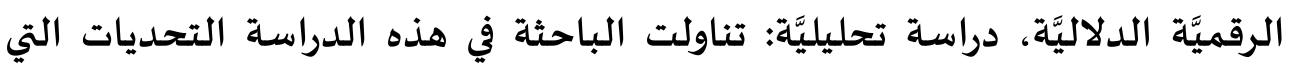

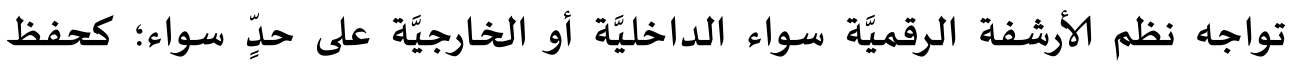

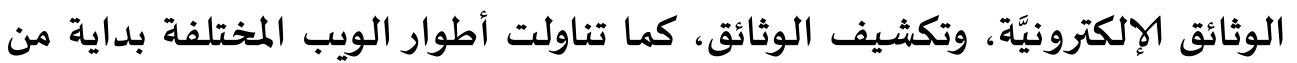

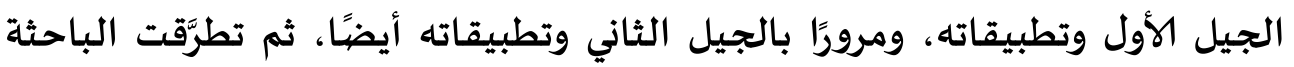

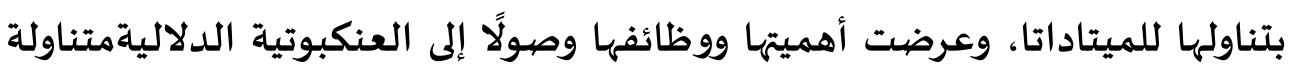

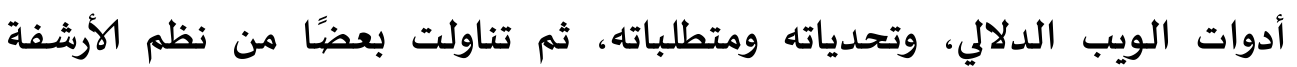

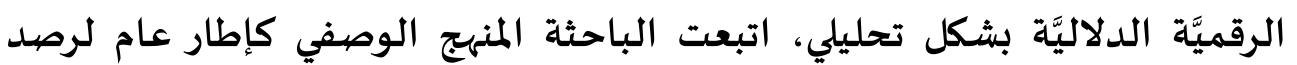
وجمع البيانات

الدراسة السابعة دراسة سامر العوض حسين (2016) عن (نظم المكتبات

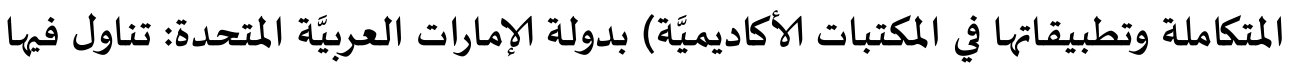

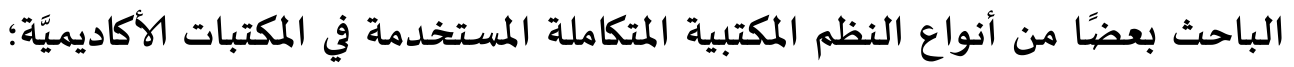

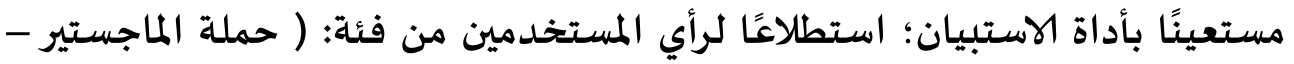
والدكتوراه)، ونسبة ضئيلة من حاملي درجة البكالوريوس، وكانت النظم عينة

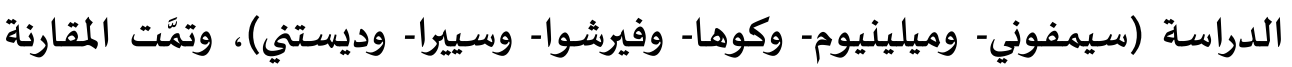
وفقًا لخمسـة معايير، هي على التوالي: 1. الدقة: دقة نسبة المعلومات الصحيحة من مجموعة المعلومات التي تمَّ تقديمها. 
2. الجدول الزمني: ستفقد المعلومات الدقيقة قيمتها إذا لم تصل إلى المتلقي في الوقت المناسب؛ حيث توجد صعوبة في تحقيق الدقة عند استخدام نظم إيصال المعلومات التقليديَّة. 3. الوضع المالي: يعتبر اقتصياد المعلومات من الأهمية بمكان عند مناقشة نظم

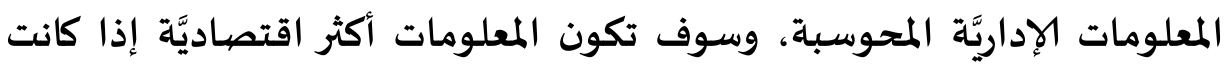

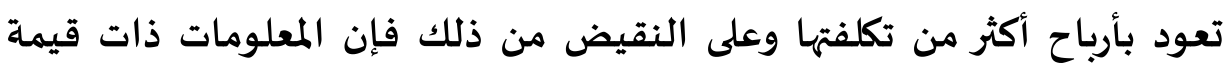

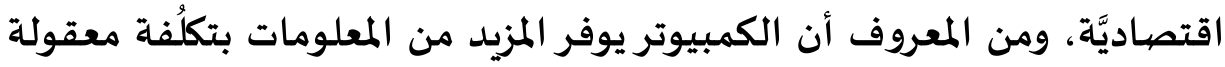
عكس النظام اليدوي التقليدي. 4. 4. التغطية الشاملة: يعني الشمول وإدراج المعلومات أو تقديم حقائق أسـاسيَّة، يطلبها المستفيدون، أو صُنََّّاع القرار.

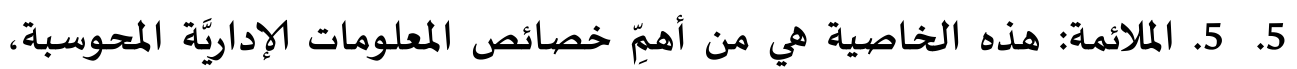

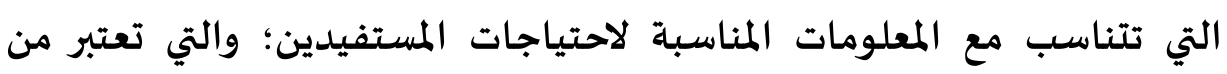

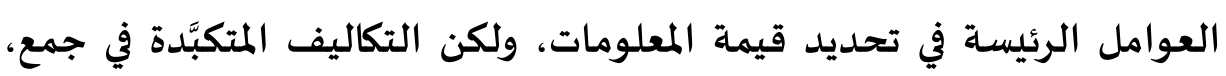

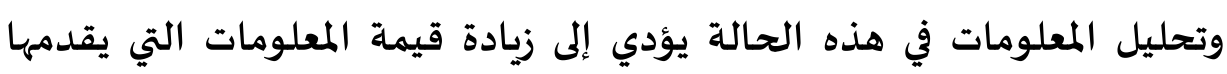
النظام، وتلبي احتياجات المستخدمين. وإذا تحدَّثنا عن أوجه التشـابه بين هذه الدراسة ودراستننا هذه فسوف نجد أن

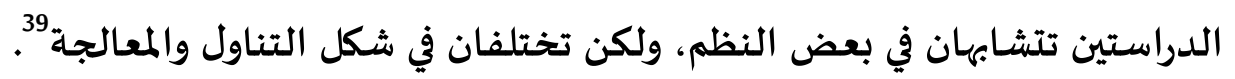

\section{النتأدجوالتوصيات}

- يمكن القول بأن اختلفت معظم الدراسـات في طرق التناول فهناك من تناولت

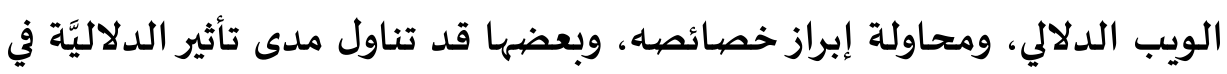

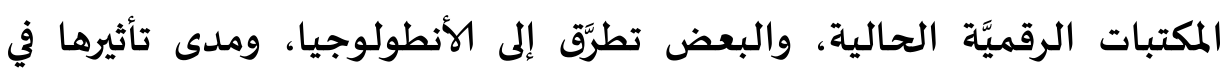
الويب الدلالي، وبعض الدراسات تناولت النظم بشكل قد يكون منفصيلًا كدراسة المالة حالة، أو تناولها بشكل عام. 
- تعدت الدراسات التي تناولت مدى تأثير الدلاليَّة على المكتبات الرقميَّة في بداية

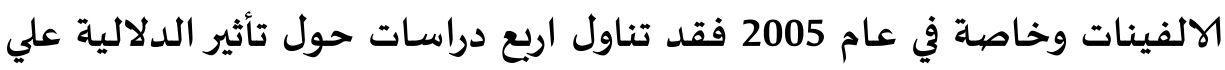

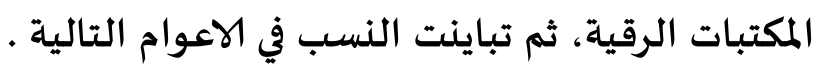

- اما عن الدراسـات التي تناولت الارتباط بين الأنطولوجيا والعنكبوتية الدلالية

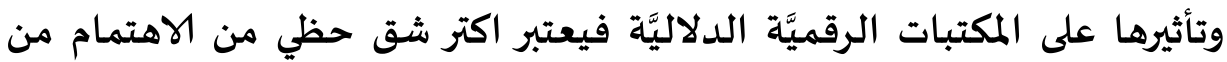

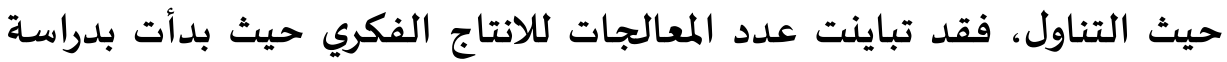

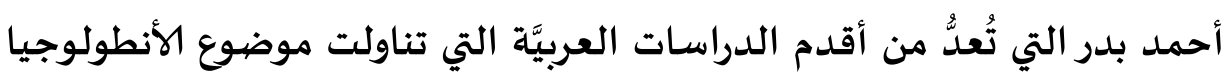

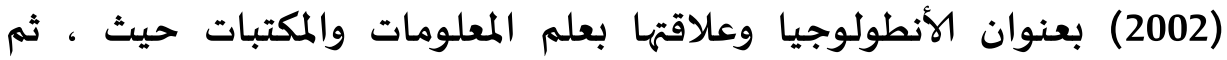

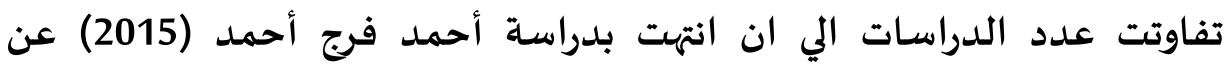

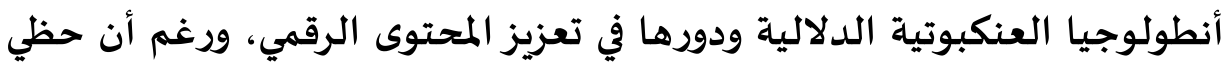
هذا الجانب بالاهتمام الا انه بفتقر إلى الدراسـات التطبيقية. - يحتاج الانتاج الفكري الي المزيد من الدراسات حول تطور نظم المكتبات الرقمية

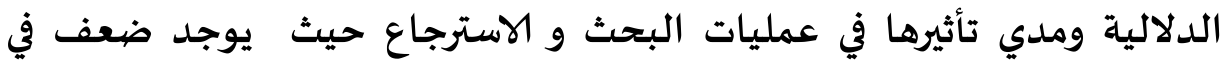
التغطية الموضوعية، فوقاً لاحصيائية الدراسات فقد تناولتها سبع درات دراسات مختلفه في طريق التناول، وقد اتفقت دراسـة كل من (هبة محمود)، ودراسة الموهية

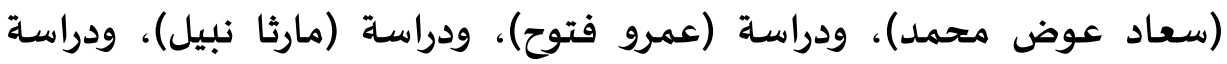

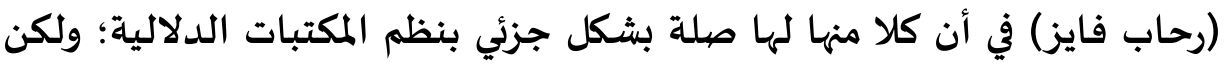

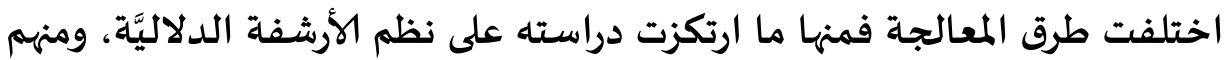

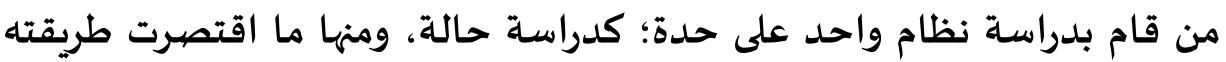

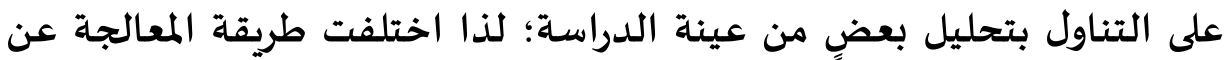
أطروحة الماجستير (التي اعتمدت على هذه المراجعة) (40)؛ حيث تمَّ مسح نظم المئه المكتبات الرقميَّة كلا من نظام (جريستون- فيدورا- دروبال- ديسبيس الميس - سييراكوها) ، ثم المقارنة باستخدام قائمة مراجعة علميَّة مقننة وفقًا لمعايير محدددة. 
1 قواعد البيانات العالمية، تمَّ الاطلاع في2020/2/19 متاح على: http://www.ekb.eg/resources?sourcesLang=ar

2 دليل دوريات الوصول الحر DOA، تمَّ الاطلاع في 2020/5/28، متاح على: https://doaj.org 3 اتحاد المكتبات الجامعيَّة المصريَّة: تمَّ الاطلاع في 2020/5/28، متاح على: http://srv4.eulc.edu.eg/eulc_v5/libraries/start.aspx

$$
4 \text { دار المنظومة، تمَّ الاطلاع في 2020/5/28، متاح على: }
$$
http://0810gwnd2.1104.y.http.search.mandumah.com.mplb.ekb.eg/Search/Results? lookfor=\%D9\%86\%D8\%B8\%D9\%85+\%D8\%A7\%D9\%84\%D9\%85\%D9\%83\%D 8\%AA\%D8\%A8\%D8\%A7\%D8\%AA+\%D8\%A7\%D9\%84\%D8\%B1\%D9\%82\%D 9\%85\%D9\%8A\%D8\%A9+\&type=AllFields\&submit=\% D8\%A7\% D8\%A8\%D8\% AD\%D8\%AB\&limit=20\&sort=relevance

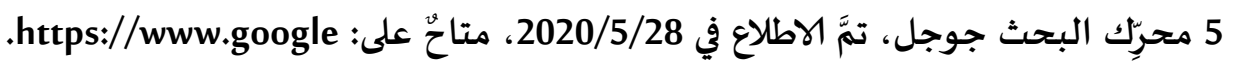

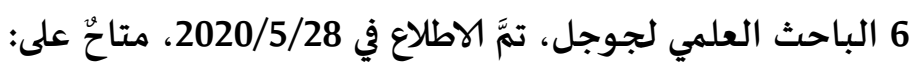
/https://scholar.google.com.eg

${ }^{7}$ Kim, Hyunki. (2005) Developing Semantic Digital Libraries Using Data Mining Techniques.- Ph.D.- University of Florida, 126 pages

$$
\text { تمَّ الاطلاع في 2020/5/29، متاح على: }
$$

http://etd.fcla.edu/UF/UFE0010105/kim_h.pdf

${ }^{8}$ Papadakis, I., Avramidis, A. and Chrissikopoulos, V. (2005) Reasoning Against

A Semantic Digital Library Framework Based On Grid Technology.- Library Management, 26 (4/5): pp. 246 - 260.-. : Available at :

http//www.emeraldinsigabs/10.1108/01435120510596099 تمَّ الاطلاع في 2020/5/29 
${ }^{9}$ Warren, P. and Alsmeyer, D. (2005) Applying Semantic Technology To A Digital Library: A Case Study.- Library Management, 26 (4/5): pp. 196 - 205.- Available at: http://www.emeraldinsight.com/doi/abs/10.1108/01435120510596053

${ }^{10}$ Sicilia, M. and Davies, J. (2005) Digital Libraries In The Knowledge Era:

Knowledge Management And Semantic Web Technologies.- Library

Management, 26 (4/5): pp.170 - 175.- Available at:

http://www.emeraldinsight.com/journals.htm?articleid=1501534\&show=html

11 عبد الحميد، رجب ( 2007): تقنيات الويب الدلالي للمكتبات الرقميَّة، journal http://www.journal.cybrarians.info/index.php?option=com_content\&view=arti cle\&id=417:2009-08-02-07-51-38\&catid=137:2009-05-20-09-51-17 ${ }^{12}$ Burke, Mary. (2009) The Semantic Web And The Digital Library.- Aslib Proceedings, 61 (3): pp. 316 - 322.- Available at:

http://www.emeraldinsight.com/journals.htm?issn=0001$253 \times$ \&volume $=61$ \&issue $=3$ \&articleid $=1793340 \&$ show $=$ html مصطفي، حسام العباسي. (2009): الويب الدلالي semanti web وعلاقته باسترجاع المعلومات، ثورة المعلومات والتحديات مصر الحديثةة، تمَّ الاطلاع في 2019/1/1، متاح على: http://alabbassyblogger.blogspot.com.eg/2009/10/semantic-web.html 14 مراد، غسان؛ وبشير، عماد (2010)، الرصد الاستراتيجي للمعلومات، الأساليب الحديثة للبحث عن المعلومات والويب الدلالي، (ندوة) 2010/6/3م، المركز الاستشـاري للدراسـات والتوثيق، تارتخ الاطلاع 2017/5/29. 15 الهزاني، نورة بنت ناصهر، مفهوم وبنية الويب الدلالي، المعلوماتية - السعودية، ع ع عاريق الإع

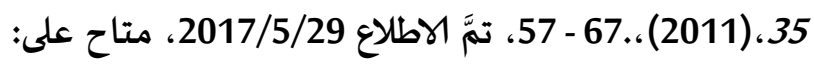
http://0810gwrvm.1104.y.http.content.mandumah.com.mplb.ekb.eg/downloa d?t=fdeb4143b0f284951e49d8d86601f245fcf16726\&f=IUA4FQLhk9x4HoQXNe gYiKyRP8c2ML6K7CkXXkD6pjE=\&s=1 
${ }^{16}$ نشرتي، مؤمن سيد (2011) : الشبكة العنكبوتيَّة الدلاليَّة: هوية تبحث عن الوجود:

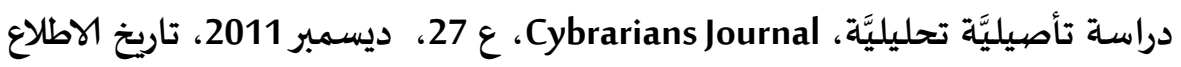

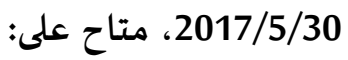

http://www.journal.cybrarians.org/index.php?option=com_content\&view=artic -le\&id=603:2011-12-01-22-24-43\&catid=253:2011-11-28-21-19 17 مؤمن سيد نشرتي: محركات البحث الدلاليَّة على الشبكة العنكبوتيَّة: دراسة مسحيَّة

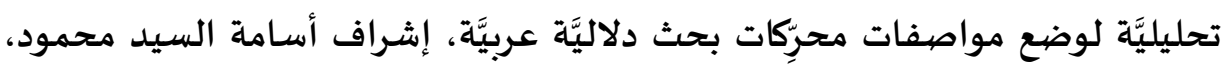

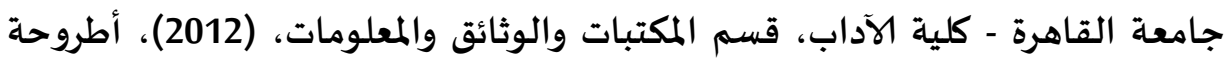
ماجستير. 18 أوكلبي، على بن ذيب (2012): تطبيقات الويب الدلالي في بيئة المعرفة، مجلة مكتبة الملك

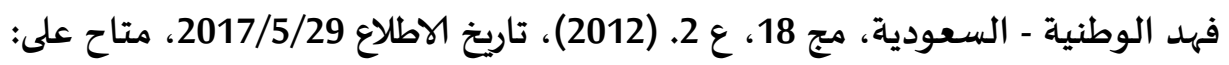
http://0810g7c56.1103.y.http.content.mandumah.com.mplb.ekb.eg/download $\underline{? t=74271331 c b 44 b 725209 d 011717 a d 89 a 3 d 5 e a 6924 \& f=q w 6 h K t \mid 2 y s q h R 4 W N U v}$

bDrTpvfJu0gljDtL4QsflKhGQ=\&s=1

19 نرمين إبراهيم على اللبان: تطبيقات الويب الدلالي في مجال الوثائق والوشيف، دراسة

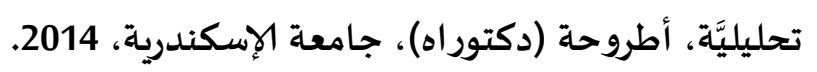

20 خibraries

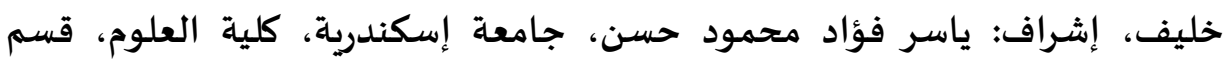

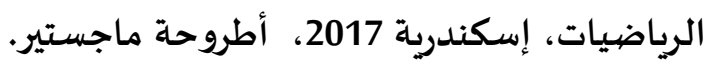

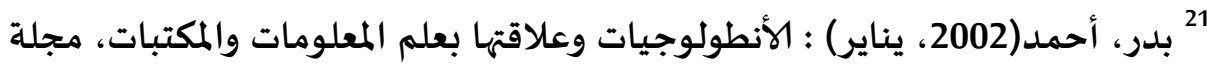

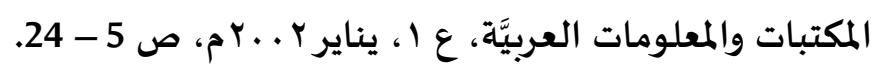

22 Pampati. Phanindra. Adding Ontologies to Graph- based Data Mining-.

Texas: P. Pampati, 2003.- 69 p. Thesis (Ph.D.). The University of Texas at Arlington 
${ }^{23}$ Web Qin,li. Change detection and management for the semaintic web. Proquest dissertations And theses. (Ph.D.dissertation). Rutgers the state university of jersey, 2005.

${ }^{24}$ JRG Pulid. IDENTIFYING ONTOLOGY COMPONENTS FROM DIGITAL ARCHIVES FOR THE SEMANTIC WEB .ininternational conference, 23-26 Jenuary2006,Universtiy of Colima, $m$ exico

25 وصال إبراهيم أحمد: الأنطولوجيا ونظم إدارة المعرفة عالم، المؤتمر الحادي والعشرون

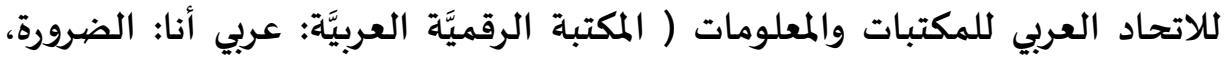

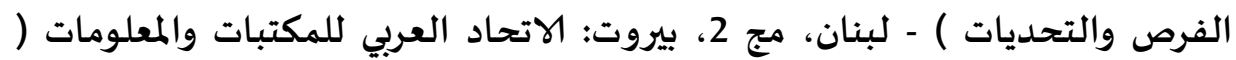
اعلم ) ووزارة الثقافة وجمعية المكتبات بالجمههورية اللبنانيَّة، 2139 - 2140.

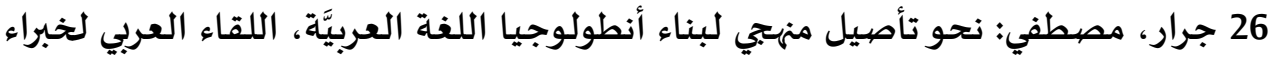

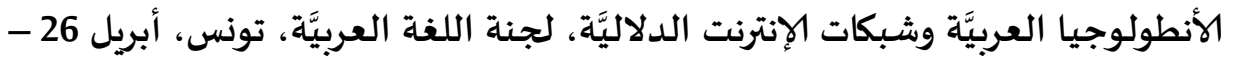

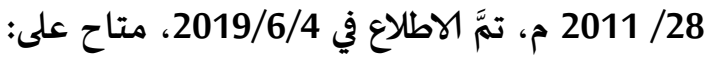
http://www.jarrar.info/publications/J11.pdf ${ }^{27}$

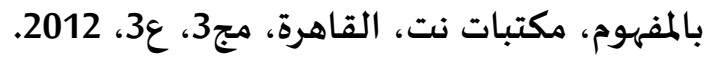

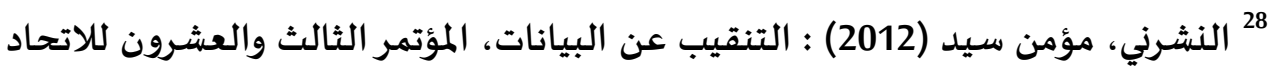

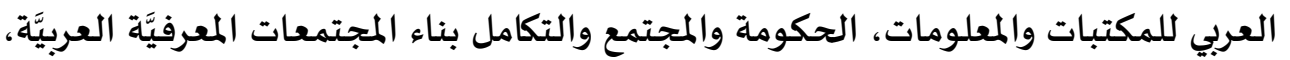

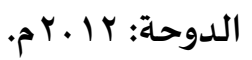
29 النشرتي، مؤمن سيد (2017): نحو التكامل المعرفي من واقع توظيف الأنطولوجيات في

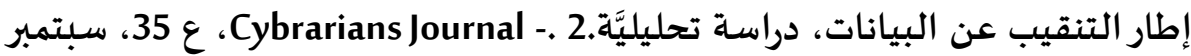

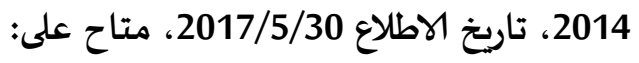
http://www.journal.cybrarians.info/index.php?option=com_content\&view=arti cle\&id=672:---------------2---\&catid=268:papers 30

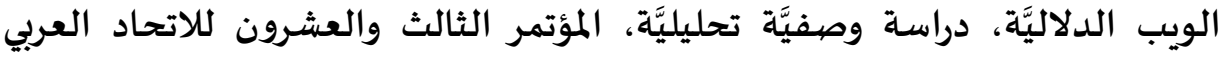
للمكتبات والمعلومات، اعلم، 2013 م. 
31 مصلوم، عبد الناصر سالم (2014): دور محررات الأنطولوجيا مفتوحة المصيدر في دعم عمليات إدارة المعرفة، جدة، جامعة الملك عبدالعزيز، أطروحة ماجستير، ملخص الدراسـة متوافر على:

https://www.kau.edu.sa/Show_Res.aspx?Site_ID=306\&LNG=AR\&RN=66013 32 أحمد، فرج أحمد (2013) : أنطولوجيا الويب الدلالي ودورها في تعزيز المحتوى الرقمي: دراسـة المفاهيم والبنية الهيكليَّة والخدمات التفاعليَّة في البوابات الدلاليَّة للتعلم الإلكتروني، مجلة المكتبات والمعلومات العربيَّة، ع4، أكتوبر 2015، تارتخ الاطلاع2019/6/3ttps:/www.slideshare.net/egyptien721/ss- متاح على:

عواطف على مكاوي: أسـاسيات البرمجيات والنظم مفتوحة المصيدر ( O.S.S.)، دراسـة تحليليَّة لتعريفها وتاريخها ومزاياها وعيوبها ومشروعاتها ومدى أهميتها للدول النامية بما فهها مصر والهند، الاتجاهات الحديثة في المكتبات والمعلومات، 7-2006، 29،28ص. 34 فايز، رحاب. (2011): نظم المكتبات الرقميَّة الدلاليَّة، دراسة تحليليَّة مقارنة، مجلة اعلم، 9ع،10ع، [pdf] تمَّ الاطلاع في 2016/7/29. تم الاسترجاع من الرابط https://www.researchgate.net/publication/279449478_nzm_almktbat_alrqmyt aldlalyt_drast_thlylyt_mqarnt 35 عمرو فتوح حسن: تقييم نظام Greenstone من خلال بناء مكتبة رقميَّة للرسـائل الجامعيَّة المجازة للباحثين في مجال المكتبات والمعلومات بالجامعات المصريَّة، إشراف: محمد فتحي عبد الهادي، إشراف: عاطف السيد قاسم، جامعة المنوفية، كلية الآداب،

$$
\text { قسم المكتبات والمعلومات، المنوفية 2010، (أطروحة ماجستير). }
$$

36 سعاد عوض محمد حسين: Applying Semantic Web Technology in Digital Libraries = تطبيق تكنولوجيا الويب الدلالي في المكتبة الرقميَّة، إشراف محمد حامد خليف، إشراف: أحمد شريف، إشراف: محمد محمد الشنديدي، جامعة إسكندرية، كلية العلوم · قسم حاسب آلي · إسكندرية 2013، أطروحة ماجستير. 37 خفاجة، أحمد ماهر: البرمجيات مفتوحة المصيدر للمكتبات ومراكز المعلومات: معايير مقترحة لاختيار نظام مفتوح المصدر لإدارة المكتبات العربيَّة، CCybrarians Journal، ع 36، 


$$
\text { ديسمبر 2014، تاريخ الاطلاع 2016/1/1، متاح على: }
$$

http://www.journal.cybrarians.org/index.php?option=com_content\&view=artic

le\&id=676:opensource\&catid=270:studies\&ltemid=99

38 مرثا نبيل صبري عبد السيد: نظم الأرشفة الرقميَّة الدلاليَّة : Semantic Digital

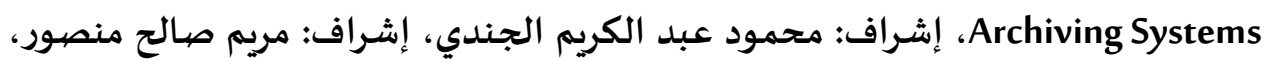

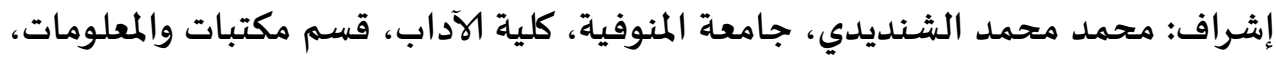
المنوفية2016، أطروحة ماجستير. 39 حسين، سـامر العوض (2016): نظم المكتبات المتكاملة وتطبيقاتها في المكتبات الأكاديميَّة بدولة الإمارات العربيَّة المتحدة.- مدونة نسيج.-- تم الاطلاع في 2020/2/2. http://blog.naseej.com/\%D8\%A7\%D9\%84\%D9\%85\%D9\%83\%D8\%AA\%D8\%A8 \%D8\%A7\%D8\%AA\%D8\%A7\%D9\%84\%D8\%A3\%D9\%83\%D8\%A7\%D8\%AF\%D9\%8A\%D9\%85\%D9\% 8A\%D8\%A9\%D8\%A7\%D9\%84\%D9\%85\%D8\%AA\%D9\%83\%D8\%A7\%D9\%85\%D9\%84\%D8\% A9-\%D9\%81\%D9\%8A\%D8\%A7\%D9\%84\%D8\%A5\%D9\%85\%D8\%A7\%D8\%B1\%D8\%A7\%D8\%AA-3/ 40 منار محمد سامي زكي. نُظم المكتبات الرقميَّة الدلاليَّة دراسة تحليليَّة مع وضيع تصور

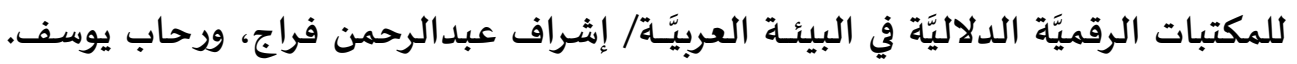
بني سويف: محافظة بني سويف، قسم علوم المعلومات، 2020. رسالة ماجستير (قيد البحث). 
